\title{
Research Article \\ Geometric Lattice Structure of Covering-Based Rough Sets through Matroids
}

\author{
Aiping Huang and William Zhu \\ Lab of Granular Computing, Zhangzhou Normal University, Zhangzhou 363000, China \\ Correspondence should be addressed to William Zhu, williamfengzhu@gmail.com
}

Received 26 August 2012; Accepted 21 November 2012

Academic Editor: Zhijun Liu

Copyright (c) 2012 A. Huang and W. Zhu. This is an open access article distributed under the Creative Commons Attribution License, which permits unrestricted use, distribution, and reproduction in any medium, provided the original work is properly cited.

Covering-based rough set theory is a useful tool to deal with inexact, uncertain, or vague knowledge in information systems. Geometric lattice has been widely used in diverse fields, especially search algorithm design, which plays an important role in covering reductions. In this paper, we construct three geometric lattice structures of covering-based rough sets through matroids and study the relationship among them. First, a geometric lattice structure of coveringbased rough sets is established through the transversal matroid induced by a covering. Then its characteristics, such as atoms, modular elements, and modular pairs, are studied. We also construct a one-to-one correspondence between this type of geometric lattices and transversal matroids in the context of covering-based rough sets. Second, we present three sufficient and necessary conditions for two types of covering upper approximation operators to be closure operators of matroids. We also represent two types of matroids through closure axioms and then obtain two geometric lattice structures of covering-based rough sets. Third, we study the relationship among these three geometric lattice structures. Some core concepts such as reducible elements in coveringbased rough sets are investigated with geometric lattices. In a word, this work points out an interesting view, namely, geometric lattice, to study covering-based rough sets.

\section{Introduction}

Rough set theory [1] was proposed by Pawlak to deal with granularity in information systems. It is based on equivalence relations. However, the equivalence relation is rather strict, hence the applications of the classical rough set theory are quite limited. For this reason, rough set theory has been extended to generalized rough set theory based on tolerance relation [2], similarity relation [3], and arbitrary binary relation [4-8]. Through extending a partition to a covering, we generalize rough set theory to covering-based rough set theory [911]. Because of its high efficiency in many complicated problems such as attribute reduction and rule learning in incomplete information/decision, covering-based rough set theory has been attracting increasing research interest $[12,13]$. 
Lattice is suggested by the form of the Hasse diagram depicting it. In mathematics, a lattice is a partially ordered set in which any two elements have a unique supremum (also called a least upper bound or join) and a unique infimum (also called a greatest lower bound or meet). They encode the algebraic behavior of the entailment relation and such basic logical connectives as "and" (conjunction) and "or" (disjunction), which results in adequate algebraic semantics for a variety of logical systems. Lattice, especially geometric lattice, is one of the most important algebraic structures and is used extensively in both theoretical and applicable fields, such as data analysis, formal concept analysis [14-16], and domain theory [17].

Matroid theory $[18,19]$ borrows extensively from linear algebra theory and graph theory. There are dozens of equivalent ways to define a matroid. Significant definitions of a matroid include those in terms of independent sets, bases, circuits, closed sets or flats and rank functions, which provide well-established platforms to connect with other theories. In applications, matroids have been widely used in many fields such as combinatorial optimization, network flows, and algorithm design, especially greedy algorithm design [20, 21]. Some works on the connection between rough sets and matroids have been conducted [22-25].

In this paper, we pay attention to geometric lattice structures of covering basedrough sets through matroids. First, a geometric lattice structure in covering-based rough sets is generated by the transversal matroid induced by a covering. Moreover, we study the characteristics of the geometric lattice structure, such as atoms, modular elements, and modular pairs. We also point out a one-to-one correspondence between this type of geometric lattices and transversal matroids in the context of covering-based rough sets. Second, generally, covering upper approximation operators are not necessarily closure operators of matroids. Then we present three sufficient and necessary conditions for two types of covering upper approximation operators to be closure operators of matroids and exhibit representations of corresponding special matroids. We study the properties of these matroids and their closed-set lattices which are also geometric lattices. Third, we study the relationship among these three geometric lattices through corresponding matroids. Furthermore, some core concepts such as reducible and immured elements in covering-based rough sets are studied by geometric lattices.

The rest of this paper is organized as follows. In Section 2, we recall some fundamental concepts related to covering-based rough sets, lattices, and matroids. Section 3 establishes a geometric lattice structure of covering-based rough sets through the transversal matroid induced by a covering. In Section 4, we present two geometric lattice structures of coveringbased rough sets through two types of upper approximation operators. Section 5 studies the relationship among these three geometric lattice structures. This paper is concluded and further work is pointed out in Section 6.

\section{Preliminaries}

In this section, we review some basic concepts of matroids, lattices, and covering-based rough sets.

\subsection{Matroids}

Matroid theory borrows extensively from the terminology of linear algebra theory and graph theory, largely because it is the abstraction of various notions of central importance in these fields, such as independent set, base, and rank function. We introduce the concept of matroid, first. 
Definition 2.1 (Matroid [19]). A matroid is an ordered pair $(E, O)$ consisting of a finite set $E$ and a collection $\supset$ of subsets of $E$ satisfying the following three conditions.

(I1) $\emptyset \in \supset$.

(I2) If $I \in \supset$ and $I^{\prime} \subseteq I$, then $I^{\prime} \in \supset$.

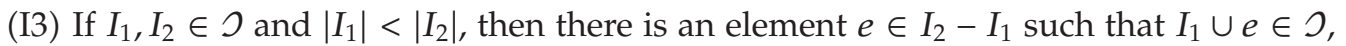
where $|X|$ denotes the cardinality of $X$.

Let $M=M(E, \supset)$ be a matroid. The members of $\supset$ are the independent sets of $M$. A set in $\supset$ is maximal, in the sense of inclusion, is called a base of the matroid $M$. If $A \notin \supset, A$ is called a dependent set of the matroid $M$. In the sense of inclusion, a minimal dependent subset of $E$ is called a circuit of the matroid $M$. If $\{a\}$ is a circuit, we call $\{a\}$ a loop. Moreover, if $\{a, b\}$ is a circuit, then $a$ and $b$ are said to be parallel. A matroid is called a simple matroid if it has no loops and no parallel elements. The rank function of a matroid is a function $r_{M}$ : $2^{E} \rightarrow N$ defined by $r_{M}(X)=\max \{|I|: I \subseteq X, I \in \supset\}(X \subseteq E)$. For each $X \subseteq E$, we say $\mathrm{cl}_{M}(X)=\left\{a \in E: r_{M}(X)=r_{M}(X \cup\{a\})\right\}$ is the closure of $X$ in $M$. When there is no confusion, we use the symbol $\mathrm{cl}(X)$ for short. $X$ is called a closure set if $\mathrm{cl}(X)=X$.

The rank function of a matroid, directly analogous to a similar theorem of linear algebra, has the following proposition.

Proposition 2.2 (Rank axiom [19]). Let $E$ be a set. A function $r_{M}: 2^{E} \rightarrow N$ is the rank function of a matroid on $E$ if and only if it satisfies the following conditions.

(R1) For all $X \in 2^{E}, 0 \leq r_{M}(X) \leq|X|$.

(R2) If $X \subseteq Y \subseteq E$, then $r_{M}(X) \leq r_{M}(Y)$.

(R3) If $X, Y \subseteq E$, then $r_{M}(X \cup Y)+r_{M}(X \cap Y) \leq r_{M}(X)+r_{M}(Y)$.

The following proposition is the closure axiom of a matroid. It means that a operator satisfies the following four conditions if and only if it is the closure operator of a matroid.

Proposition 2.3 (Closure axiom [19]). Let $E$ be a set. A function $c_{M}: 2^{E} \rightarrow 2^{E}$ is the closure operator of a matroid $M$ on $E$ if and only if it satisfies the following conditions.

(1) If $X \subseteq E$, then $X \subseteq c l_{M}(X)$.

(2) If $X \subseteq Y \subseteq E$, then $c l_{M}(X) \subseteq c l_{M}(Y)$.

(3) If $X \subseteq E, c l_{M}\left(c l_{M}(X)\right)=c l_{M}(X)$.

(4) If $X \subseteq E, x \in E$ and $y \in c l_{M}(X \cup\{x\})-c l_{M}(X)$, then $x \in c l_{M}(X \cup\{y\})$.

Transversal theory is a branch of a matroid theory. It shows how to induce a matroid, namely, transversal matroid, from a family of subsets of a set. Hence, the transversal matroid establishes a bridge between collections of subsets of a set and matroids.

Definition 2.4 (Transversal [19]). Let $S$ be a nonempty finite set and $J=\{1,2, \ldots, m\}$. $\mathcal{F}=\left\{F_{1}, F_{2}, \ldots, F_{m}\right\}$ denotes a family of subsets of $S$. A transversal or system of distinct representatives of $\left\{F_{1}, F_{2}, \ldots, F_{m}\right\}$ is a subset $\left\{e_{1}, e_{2}, \ldots, e_{m}\right\}$ of $S$ such that $e_{i} \in F_{i}$ for all $i$ in $J$. If for some subset $K$ of $J, X$ is a transversal of $\left\{F_{i}: i \in K\right\}$, then $X$ is said to be a partial transversal of $\left\{F_{1}, F_{2}, \ldots, F_{m}\right\}$. 
Example 2.5. Let $S=\{1,2,3,4\}, F_{1}=\{2,3\}, F_{2}=\{4\}$ and $F_{3}=\{2,4\}$. For $\mathcal{F}=\left\{F_{1}, F_{2}, F_{3}\right\}$, $T=\{2,3,4\}$ is a transversal of $\mathscr{F}$ because $2 \in F_{3}, 3 \in F_{1}$ and $4 \in F_{2} . T^{\prime}=\{2,4\}$ is a partial transversal of $\mathcal{F}$ because there exists a subset of $\mathcal{F}$, that is, $\left\{K_{1}, K_{2}\right\}$, such that $T^{\prime}$ is a transversal of it.

The following proposition shows what kind of matroids are transversal matroid.

Proposition 2.6 (Transversal matroid [19]). Let $\mathcal{F}=\left\{F_{i}: i \in J\right\}$ be a family subsets of $E . M(\mathcal{F})=$ $(E, \supset(\mathscr{F}))$ is a matroid, where $\supset(\mathcal{F})$ is the family of all partial transversals of $\mathscr{F}$. One calls $M(\mathscr{F})=$ $(E, \supset(\mathcal{F}))$ the transversal matroid induced by $\mathcal{F}$.

\subsection{Lattices}

Let $(P, \leq)$ be an ordered set and $a, b \in P$. We say that $a$ is covered by $b$ (or $b$ covers $a$ ) if $a<b$ and there is no element $c$ in $P$ with $a<c<b$. A chain in $P$ from $x_{0}$ to $x_{n}$ is a subset $\left\{x_{0}, x_{1}, \ldots, x_{n}\right\}$ of $P$ such that $x_{0}<x_{1}<\cdots<x_{n}$. The length of such a chain is $n$, and the chain is maximal if $x_{i}$ covers $x_{i-1}$ for all $i \in\{1,2, \ldots, n\}$. If, for every pair $\{a, b\}$ of elements of $P$ with $a<b$, all maximal chains from $a$ to $b$ have the same length, then $P$ is said to satisfy the Jordan-Dedekind chain condition. The height $h_{P}(y)$ of an element $y$ of $P$ is the maximum length of a chain from 0 to $y$. A poset $(\mathcal{L}, \leq)$ is a lattice if $a \vee b$ and $a \wedge b$ exist for all $a, b \in \mathcal{L}$. Suppose $\mathcal{L}$ is a lattice with zero element 0 . If $a$ covers 0 , then $a \in \mathcal{L}$ is called an atom of $\mathcal{L}$. Moreover, the atoms of $\mathcal{L}$ are precisely the elements of height one. It is not difficult to check that every finite lattice has a zero and the one. A finite lattice $\mathcal{L}$ is called semimodular if it satisfies the Jordan-Dedekind chain condition and for every pair $\{x, y\}$ of elements of $\mathcal{L}$, the equality $h_{\mathfrak{L}}(x)+h_{\mathfrak{L}}(y) \geq h_{\mathfrak{L}}(x \vee y)+h_{\mathfrak{L}}(x \wedge y)$ holds. A geometric lattice is a finite semimodular lattice in which every element is a join of atoms.

Next, we introduce the modular element and modular pair which are important concepts of lattices.

Definition 2.7 (see [17]). Let $\mathcal{L}$ be a lattice and $a, b \in \mathcal{L}$.

(ME) For all $x, z \in \mathcal{L}, x \geq z$ implies $x \wedge(a \vee z)=(x \wedge a) \vee z$, then $a$ is called a modular element of $\mathcal{L}$.

(MP) For all $z \in \mathcal{L}, b \geq z$ implies $b \wedge(a \vee z)=(b \wedge a) \vee z$, then $(a, b)$ is called a modular pair of $\mathcal{L}$.

As we know, if $a$ is a modular element of $\mathcal{L}$, then $(a, b)$ is a modular pair of $\mathcal{L}$ for all $b \in \mathcal{L}$, which roots in an important result of lattices. For a semimodular lattice, modular pair has close relation with height function.

Lemma 2.8 (see [17]). Let $\mathcal{L}$ be a semimodular lattice, then $(a, b)$ is a modular pair if and only if $h_{\mathcal{L}}(a \vee b)+h_{\mathcal{L}}(a \wedge b)=h_{\mathcal{L}}(a)+h_{\mathcal{L}}(b)$ for all $a, b \in \mathcal{L}$.

\subsection{Closed-Set Lattice of a Matroid}

If $M$ is a matroid and $\mathcal{L}(M)$ denotes the set of all closed sets of $M$ ordered by inclusion, then $(\mathcal{L}(M), \subseteq)$ is a lattice. In addition to that, the operations join and meet of it are, respectively, defined as $X \wedge Y=X \cap Y$ and $X \vee Y=\operatorname{cl}_{M}(X \cup Y)$ for all $X, Y \in \mathcal{L}(M)$. The zero of $\mathcal{L}(M)$ 
is $\mathrm{cl}_{M}(\emptyset)$, while the one is $E$. The following lemma gives another definition of a geometric lattice from the viewpoint of matroid. In fact, the set of all closed sets of a matroid ordered by inclusion is a geometric lattice.

Lemma 2.9 (see [19]). A lattice $\mathcal{\perp}$ is geometric if and only if it is the lattice of closed sets of a matroid.

The following lemma establishes the relation between the rank function of a matroid and the height function of the closed-set lattice of the matroid.

Lemma 2.10 (see [19]). Let $M$ be a matroid. $h_{\mathcal{L}(M)}(X)=r_{M}(X)$ for all $X \in \mathcal{L}(M)$.

\subsection{Covering-Based Rough Sets}

In this subsection, we introduce some concepts of covering-based rough sets used in this paper.

Definition 2.11 (Covering and partition). Let $E$ be a universe of discourse, $C$ a family of subsets of $E$, and none of subsets in $\mathcal{C}$ be empty. If $\cup \mathcal{C}=E$, then $\mathcal{C}$ is called a covering of $E$. Any element of $\mathcal{C}$ is called a covering block. If $D$ is a covering of $E$ and it is a family of pairwise disjoint subsets of $E$, then $P$ is called a partition of $E$.

It is clear that a partition of $E$ is certainly a covering of $E$, so the concept of a covering is an extension of the concept of a partition.

Let $E$ be a finite set and $R$ be an equivalent relation on $E$. $R$ will generate a partition $E / R=\left\{Y_{1}, Y_{2}, \ldots, Y_{m}\right\}$ of $E$, where $Y_{1}, Y_{2}, \ldots, Y_{m}$ are the equivalence classes generated by $R$. For all $X \subseteq E$, the lower and upper approximations of $X$, are, respectively, defined as follows:

$$
\begin{gathered}
R_{*}(X)=\bigcup\left\{Y_{i} \in \frac{E}{R}: Y_{i} \subseteq X\right\}, \\
R^{*}(X)=\bigcup\left\{Y_{i} \in \frac{E}{R}: Y_{i} \bigcap X \neq \emptyset\right\} .
\end{gathered}
$$

Next, we introduce certain important concepts of covering-based rough sets, such as minimal description, indiscernible neighborhood, neighborhood, reducible element, and approximation operators.

Definition 2.12 (Minimal description [26]). Let $\mathcal{C}$ be a covering of $E$ and $x \in E$ :

$$
M d_{\mathcal{C}}(x)=\{K \in \mathcal{C}: x \in K \wedge(\forall S \in \mathcal{C} \wedge x \in S \wedge S \subseteq K \Longrightarrow K=S)\}
$$

is called the minimal description of $x$. When the covering is clear, we omit the lowercase $\mathcal{C}$ in the minimal description.

Definition 2.13 (Indiscernible neighborhood and neighborhood [27, 28]). Let $(E, \mathcal{C})$ be a covering approximation space and $x \in E$.

$\cup\{K: x \in K \in \mathcal{C}\}$ is called the indiscernible neighborhood of $x$ and denoted as $I_{\mathcal{C}}(x)$. 
$\cap\{K: x \in K \in \mathcal{C}\}$ is called the neighborhood of $x$ and denoted as $N_{\mathcal{C}}(x)$. When the covering is clear, we omit the lowercase $\mathcal{C}$.

Definition 2.14 (A reducible covering [29]). Let $\mathcal{C}$ be a covering of a domain $E$ and $K \in \mathcal{C}$. If $K$ is a union of some sets in $\mathcal{C}-\{K\}$, we say $K$ is a reducible element in $\mathcal{C}$; otherwise $K$ is an irreducible element in $\mathcal{C}$. If every element in $\mathcal{C}$ is irreducible, we say $\mathcal{C}$ is irreducible; otherwise $\mathcal{C}$ is reducible.

Definition 2.15 (Reduct [29]). For a covering $\mathcal{C}$ of a universe $E$, when we remove all reducible elements from $C$, the set of remaining elements is still a covering of $E$, and this new irreducible covering has not reducible element. We call thus new covering a reduct of $\mathcal{C}$ and it is denoted by $\operatorname{reduct}(\mathcal{C})$.

Definition 2.16 (Immured element [27]). Let $\mathcal{C}$ be a covering of $E$ and $K$ an element of $\mathcal{C}$. If there exists another element $K^{\prime}$ of $\mathcal{C}$ such that $K \subset K^{\prime}$, we say that $K$ is an immured element of covering $\mathcal{C}$.

Definition 2.17 (Exclusion [27]). Let $\mathcal{C}$ be a covering of $E$. When we remove all immured elements from $\mathcal{C}$, the set of all remaining elements is still a covering of $E$, and this new covering has no immured element. We called this new covering an exclusion of $\mathcal{C}$, and it is denoted by exclusion $(\mathcal{C})$.

The second type of covering rough set model was first studied by Pomykała in [30]. While the sixth type of covering-based upper approximation operator was first defined in [31].

Definition 2.18. Let $\mathcal{C}$ be a covering of $E$. The covering upper approximation operators $\mathrm{SH}, \mathrm{XH}: 2^{E} \rightarrow 2^{E}$ are defined as follows: For all $\mathrm{X} \in 2^{E}$,

$$
\begin{aligned}
& \mathrm{SH}(X)=\cup\{K \in \mathcal{C}: K \cap X \neq \emptyset\}=\cup\{I(x): x \in X\}, \\
& \mathrm{XH}(X)=\{x: N(x) \cap X \neq \emptyset\} .
\end{aligned}
$$

$\mathrm{SH}_{\mathcal{C}}$ and $\mathrm{XH}_{\mathcal{C}}$ are called the second and the sixth covering upper approximation operators with respect to the covering $\mathcal{C}$, respectively. When there is no confusion, we omit $\mathcal{C}$ at the lowercase.

\section{A Geometric Lattice Structure of Covering-Based Rough Sets through Transversal Matroid}

As we know, if $M$ is a matroid and $\mathcal{L}(M)$ denotes the set of all closed sets of $M$ ordered by inclusion, then $\mathcal{L}(M)$ is a geometric lattice. In this section, we study the properties such as atoms, modular elements and modular pairs of this type of geometric lattice through transversal matroid induced by a covering. We also study the structure of matroid induced by the geometric lattice. It is interesting to find that there is a one-to-one correspondence between this type of geometric lattices and transversal matroids in the context of coveringbased rough sets.

Let $E$ be a nonempty finite set and $C$ a covering of $E$. As shown in Proposition 2.6, $M(\mathcal{C})=(E, \supset(\mathcal{C}))$ is the transversal matroid induced by covering $\mathcal{C} . \mathcal{L}(M(\mathcal{C}))$ is the set of all closed sets of $M(\mathcal{C})$. Especially, $\mathcal{L}(M(D))$ is the set of all closed sets of the transversal 
matroid induced by partition $D$. Based on Lemma 2.9, we know $\mathcal{L}(M(\mathcal{C}))$ and $\mathcal{L}(M(D))$ are geometric lattices.

The theorem below connects a covering $\mathcal{C}$ with $\operatorname{cl}_{M(\mathcal{C})}(\emptyset)$. In fact, $\emptyset \in \mathcal{L}(M(\mathcal{F}))$ if and only if $\mathcal{F}$ is a covering.

Theorem 3.1. Let $E$ be a set and $M(\mathcal{F})$ a transversal matroid induced by a family of $\mathcal{F}=$ $\left\{F_{1}, F_{2}, \ldots, F_{m}\right\}$ subsets of $E$, where $F_{i} \neq \emptyset(1 \leq i \leq m) . c l_{\mp}(\emptyset)=\emptyset$ if and only if $\mathcal{F}$ is a covering of $E$.

Proof. " $\Leftarrow$ ": According to the definition of transversal matroid, any partial transversal is an independent set of transversal matroid. Since $\mathcal{F}$ is a covering, any single-point set is an independent set. Based on the definition of closure operator of a matroid, we have $\mathrm{cl}_{M(\mathcal{F})}(\emptyset)=\emptyset$.

" $\Rightarrow$ ": Since $\mathrm{cl}_{M(\mp)}(\emptyset)=\emptyset$, any single-point set is an independent set, that is, for all $x \in E$, there exists $i_{x} \in\{1,2, \ldots, m\}$ such that $x \in F_{i_{x}} \subseteq E$. Hence, $E=\bigcup_{x \in E}\{x\} \subseteq \bigcup_{x \in E} F_{i_{x}} \subseteq$ $\bigcup_{i=1}^{m} F_{i} \subseteq E$. Thus $\bigcup_{i=1}^{m} F_{i}=E$. For all $i \in\{1,2, \ldots, m\}, F_{i} \neq \emptyset$ and $\bigcup_{i=1}^{m} F_{i}=E$, hence $\mathcal{F}$ is a covering.

Theorem 3.1 indicates that the zero of $\mathcal{L}(M(\mathcal{C}))$ is $\emptyset$. The following lemma presents the form of the atoms of $\mathcal{L}(M(\mathcal{C}))$. In fact, the closure of any single-point set is an atom of the lattice.

Lemma 3.2. Let $\mathcal{C}$ be a covering of $E$. For all $x \in E, c l_{M(\mathcal{C})}(\{x\})$ is an atom of $\mathcal{L}(M(\mathcal{C}))$.

Proof. Since $C$ is a covering, we know any single-point set is an independent set. Thus for all $x \in E, r_{M(\mathcal{C})}\left(\mathrm{cl}_{M(\mathcal{C})}(\{x\})\right)=r_{M(\mathcal{C})}(\{x\})=1$. As we know, the atoms of a lattice are precisely the elements of height one. Combining with Lemma 2.10 and the fact that $\mathrm{cl}_{M(\mathcal{C})}(\{x\})$ is a closed set, we know $\mathrm{cl}_{M(\mathcal{C})}(\{x\})$ is an atom of $\mathcal{L}(M(\mathcal{C}))$.

Lemma 3.2 does not establish the concrete form of $\mathrm{cl}_{M(\mathcal{C})}(\{x\})$. In order to solve that problem, we first define two sets as follows.

Definition 3.3. Let $\mathcal{C}=\left\{K_{1}, K_{2}, \ldots, K_{m}\right\}$ be a covering of a finite set $E=\left\{x_{1}, x_{2}, \ldots, x_{n}\right\}$. We define the following.

(i) $A=\left\{K_{i}-\bigcup_{j=1, j \neq i}^{m} K_{j}: K_{i}-\bigcup_{j=1, j \neq i}^{m} K_{j} \neq \emptyset, i \in\{1,2, \ldots, m\}\right\}=\left\{A_{1}, A_{2}, \ldots, A_{s}\right\}$.

(ii) $B=E-\bigcup_{i=1}^{s} A_{i}$.

Remark 3.4. For all $i \in\{1,2, \ldots, s\}$ and $x \in A_{i}$, there exists only one block such that $x$ belongs to it, and there exist at least two blocks such that $y$ belongs to them for all $y \in B$.

The following two propositions establish two characteristics of $A$ and $B$.

Proposition 3.5. Let $\mathcal{C}$ be a covering of $E .\left\{A_{1}, A_{2}, \ldots, A_{s}\right\} \cup\{\{x\}: x \in B\}$ forms a partition of $E$.

Proof. Let $P=\left\{A_{1}, A_{2}, \ldots, A_{s}\right\} \cup\{\{x\}: x \in B\}$. According to Definition 3.3, we know $\left(\bigcup_{i=1}^{s} A_{i}\right) \cup(\cup\{\{x\}: x \in B\})=E$. Now we need to prove for all $P_{1}, P_{2} \in P, P_{1} \cap P_{2}=\emptyset$. According to the definition of $A$, if $P_{1}, P_{2} \in A$, then $P_{1} \cap P_{2}=\emptyset$. If $P_{1}, P_{2} \in\{\{x\}: x \in B\}$, then $P_{1} \cap P_{2}=\emptyset$ because $P_{i}$ and $P_{j}$ are different single-points. If $P_{1} \in A$ and $P_{2} \in\{\{x\}: x \in B\}$, then $P_{1} \cap P_{2}=\emptyset$ because $B \cap\left(\bigcup_{k=1}^{S} A_{k}\right)=\emptyset, P_{1} \subseteq \bigcup_{k=1}^{S} A_{k}$ and $P_{2} \subseteq B$. 
Proposition 3.6. $C$ is a partition of $E$ if and only if $B=\emptyset$.

Proof. According to the definition of $A$ and $B$, the necessity is obvious. Now we prove the sufficiency. If $\mathcal{C}$ is not a partition, then there exist $K_{i}, K_{j} \in \mathcal{C}$ such that $K_{i} \cap K_{j} \neq \emptyset$. Thus there exists $x \in E$ such that $x \in K_{i} \cap K_{j}$, that is, there exist at least $K_{i}, K_{j} \in \mathcal{C}$ such that $x$ belongs to them, hence $x \in B$. That contradicts the assumption that $B=\emptyset$.

Based on Lemma 3.2 and Definition 3.3, we can establish the concrete form of the atoms of lattice $\mathcal{L}(M(\mathcal{C}))$.

Theorem 3.7. Let $\mathcal{C}$ be a covering of $E .\left\{A_{1}, A_{2}, \ldots, A_{s}\right\} \cup\{\{x\}: x \in B\}$ is the set of atoms of lattice $\mathcal{L}(M(\mathcal{C}))$.

Proof. According to the definition of $A_{i}$, we may as well suppose $A_{i}=K_{h}-\bigcup_{j=1, j \neq h}^{m} K_{j}$. Based on $C$ being a covering and the definition of transversal matroid, we know any single-point set is an independent set, thus for all $x \in A_{i},\{x\}$ is an independent set. For all $y \in A_{i}$ and $y \neq x$, we know $x, y \in K_{h}$ and $x, y \notin K_{j}$ for all $1 \leq j \leq m$ and $j \neq h$, thus $x$ and $y$ cannot be chosen from different blocks in the covering $\mathcal{C}$. That shows that $\{x, y\}$ is not an independent set according to the definition of transversal matroid. Hence, $\{x\}$ is a maximal independent set included in $A_{i}$, that is, $r_{M(\mathcal{C})}\left(A_{i}\right)=1$. Next, we need to prove $A_{i}$ is a closed set. Since $A_{i} \subseteq \mathrm{cl}_{M(\mathcal{C})}\left(A_{i}\right)$, we need to prove $\mathrm{cl}_{M(\mathcal{C})}\left(A_{i}\right) \subseteq A_{i}$, that is, $y \notin A_{i}$ implies $y \notin \mathrm{cl}_{M(\mathcal{C})}\left(A_{i}\right)$. If $y \notin A_{i}$, based on the fact that $\mathcal{C}$ is a covering and the definition of $A_{i}$, then there exists $j \neq h$ such that $y \in K_{j}$. Thus $\{x, y\}\left(x \in A_{i}\right)$ is an independent set. That implies $y \notin \operatorname{cl}_{M(\mathcal{C})}\left(A_{i}\right)$, thus $\mathrm{cl}_{M(\mathcal{C})}\left(A_{i}\right)=A_{i}$. Hence, $A_{i} \in \mathcal{L}(M(\mathcal{C}))$. Combining with $r_{M(\mathcal{C})}\left(A_{i}\right)=1$, we know for all $1 \leq i \leq m, A_{i}$ is an atom of lattice $\mathcal{L}(M(\mathcal{C}))$.

According to the definition of transversal matroid and the fact that $\mathcal{C}$ is a covering, any single-point set is an independent set. Thus for all $x \in B, r_{M(\mathcal{C})}(\{x\})=1$. For all $y \in E$ and $y \neq x$, if $y \in B$, then there exist at least two blocks containing $y$ according to the definition of $B$. We may as well suppose $y \in K_{k}, K_{t}$ and $x \in K_{l}, K_{p}$, where $\left\{K_{k}, K_{t}\right\}$ may be the same as $\left\{K_{l}, K_{p}\right\}$. Based on this, $\{x, y\}$ is an independent set. This implies $y \notin \mathrm{cl}_{M(\mathcal{C})}(\{x\})$. If $y \notin B$, then we may as well suppose $y \in A_{i}$, thus $y \in K_{h}$ for the definition of $A_{i}$, where $K_{h}$ may be the same with $K_{l}$ or $K_{p}$. Based on this, $x$ and $y$ can be chosen from different blocks in covering $\mathcal{C}$, thus $\{x, y\}$ is an independent set. That implies $y \notin \mathrm{cl}_{M(\mathcal{C})}(\{x\})$. From above discussion, we have $\mathrm{cl}_{M(\mathcal{C})}(\{x\})=\{x\}$. Hence, $\{x\} \in \mathcal{L}(M(\mathcal{C}))$ for all $x \in B$. Combining with $r_{M(\mathcal{C})}(\{x\})=1$, we know $\{x\}$ is an atom of lattice $\mathcal{L}(M(\mathcal{C})$ ) for all $x \in B$.

Next, we will prove the set of atoms of lattice $\mathcal{L}(M(\mathcal{C}))$ cannot be anything but $\left\{A_{1}, A_{2}, \ldots, A_{s}\right\} \cup\{\{x\}: x \in B\}$. According to Lemma 3.2, we know $\left\{\operatorname{cl}_{M(\mathcal{C})}(\{x\}): x \in E\right\}$ is the set of atoms of lattice $\mathcal{L}(M(\mathcal{C}))$. Similar to the proof of the second part, we know that if $x \in B$ then $\operatorname{cl}_{M(\mathcal{C})}(\{x\})=\{x\}$. If $x \notin B$, then $x$ belongs to one of elements in $A$. We may as well suppose $x \in A_{i}$. Combining $A_{i}$ is an atom with $\emptyset \subseteq \mathrm{cl}_{M(\mathcal{C})}(\{x\}) \subseteq \mathrm{cl}_{M(\mathcal{C})}\left(A_{i}\right)=A_{i}$, we have $\mathrm{cl}_{M(\mathcal{C})}(\{x\})=A_{i}$. Hence, $\left\{A_{1}, A_{2}, \ldots, A_{s}\right\} \cup\{\{x\}: x \in B\}$ is the set of atoms of lattice $\mathcal{L}(M(\mathcal{C}))$.

The proposition below connects simple matroid and the cardinal number of $A_{i}$. In fact, a matroid is simple if and only if $\left|A_{i}\right|=1$ for all $A_{i} \in A$.

Lemma 3.8. Let $\mathcal{C}$ be a covering of $E$. For all $A_{i} \in A$, if $\left|A_{i}\right| \geq 2$, then $x$ and $y$ are parallel in $M(\mathcal{C})$ for all $x, y \in A_{i}$. 
Proof. According to the definition of $A_{i}$, we may as well suppose $A_{i}=K_{h}-\bigcup_{j=1, j \neq h}^{m} K_{j}$, where $1 \leq h \leq m$. For all $x, y \in A_{i}$, then $x, y \in K_{h}$, and for all $j \in\{1,2, \ldots, m\}$ and $j \neq h$, we have $x \notin K_{j}$ and $y \notin K_{j}$. Thus $\{x, y\}$ is not an independent set. Based on the definition of transversal matroid and the fact that $\mathcal{C}$ is a covering, any single-point set is an independent set. Thus $\{x\}$ or $\{y\}$ is an independent set. Hence, $x, y$ are parallel in $M(\mathcal{C})$.

Proposition 3.9. Let $\mathcal{C}$ be a covering of $E . M(\mathcal{C})$ is a simple matroid if and only if $\left|A_{i}\right|=1$ for all $A_{i} \in A$.

Proof. " $\Rightarrow$ ": Since $M(\mathcal{C})$ is a simple matroid, it does not contain parallel elements. If there exists $A_{i} \in A$ such that $\left|A_{i}\right| \neq 1$, then $\left|A_{i}\right| \geq 2$ because $A_{i} \neq \emptyset$. According to Lemma 3.8, we know for all $x, y \in A_{i}, x, y$ are parallel which contradicts the assumption that $M(\mathcal{C})$ is a simple matroid. Hence, for all $A_{i} \in A,\left|A_{i}\right|=1$.

" $\Leftarrow$ ": According to the definition of parallel element, if $\left|A_{i}\right|=1$ for all $A_{i} \in A$, then $M(\mathcal{C})$ does not contain parallel elements; otherwise, we may as well suppose $x, y$ are parallel, then there exists only one block which contains $x, y$. Hence, there exists $A_{i} \in A$ such that $x, y \in A_{i}$, that is, $\left|A_{i}\right| \geq 2$. This contradicts the fact that $\left|A_{i}\right|=1$ for all $A_{i} \in A$. Based on the definition of transversal matroid and the fact that $\mathcal{C}$ is a covering, any single-point set is an independent set, thus $M(\mathcal{C})$ does not contain loops. Hence, $M(\mathcal{C})$ does not contain parallel elements and loops which implies that $M(\mathcal{C})$ is a simple matroid.

When a covering degenerates into a partition, we also have the above results.

Corollary 3.10. Let $D=\left\{P_{1}, P_{2}, \ldots, P_{m}\right\}$ be a partition of $E$. $P$ is the set of atoms of lattice $\mathcal{L}\left(M_{p}\right)$.

Corollary 3.11. Let $D=\left\{P_{1}, P_{2}, \ldots, P_{m}\right\}$ be a partition of $E . M(D)$ is a simple matroid if and only if $\left|P_{i}\right|=1$ for all $P_{i} \in P$.

For a geometric lattice $\mathcal{L}(M(\mathcal{C}))$, any closure of single-point is an atom of it. However, the closure of any two elements of $E$ may not be a element which covers some atoms of this lattice. The following proposition shows in what condition $\mathrm{cl}_{M(\mathcal{C})}(\{x, y\})$ covers certain atoms of lattice $\mathcal{L}(M(\mathcal{C}))$.

Proposition 3.12. Let $\mathcal{C}$ be a covering of E. For all $x, y \in E, c l_{M(\mathcal{C})}(\{x, y\})$ covers $c l_{M(\mathcal{C})}(\{x\})$ if and only if there does not exist $A_{i} \in A$ such that $x, y \in A_{i}$.

Proof. " $\Leftarrow$ ": For all $x, y \in E,\{x\} \subseteq\{x, y\}$, then $\operatorname{cl}_{M(\mathcal{C})}(\{x\}) \subseteq \operatorname{cl}_{M(\mathcal{C})}(\{x, y\})$ and $1=$ $r_{M(\mathcal{C})}\left(\mathrm{cl}_{M(\mathcal{C})}(\{x\})\right) \leq r_{M(\mathcal{C})}\left(\mathrm{cl}_{M(\mathcal{C})}(\{x, y\})\right)=r_{M(\mathcal{C})}(\{x, y\}) \leq|\{x, y\}|=2$. Now we need to prove $r_{M(\mathcal{C})}\left(\mathrm{cl}_{M(\mathcal{C})}(\{x, y\})\right)=2$. If $r_{M(\mathcal{C})}\left(\mathrm{cl}_{M(\mathcal{C})}(\{x, y\})\right)=r_{M(\mathcal{C})}(\{x, y\})=1=r_{M(\mathcal{C})}(\{x\})$, then $\{x, y\} \notin \supset(\mathcal{C})$, that is, there is only one block contains $x, y$. It means that there exists $A_{i} \in A$ such that $x, y \in A_{i}$. That contradicts the hypothesis. Hence, $r_{M(\mathcal{C})}\left(\mathrm{cl}_{M(\mathcal{C})}(\{x, y\})\right)=2$, that is, $\mathrm{cl}_{M(\mathcal{C})}(\{x, y\})$ covers $\mathrm{cl}_{M(\mathcal{C})}(\{x\})$.

" $\Rightarrow$ ": For all $x, y \in E$, if there exists $A_{i} \in A$ such that $x, y \in A_{i}$, then there is only one block contains $x, y$, thus $x, y \notin \supset(\mathcal{C})$, hence $\{x, y\} \subseteq \mathrm{cl}_{M(\mathcal{C})}(\{x\})$. That implies $\mathrm{cl}_{M(\mathcal{C})}(\{x, y\}) \subseteq$ $\mathrm{cl}_{M(\mathcal{C})}(\{x\})$ which contradicts the assumption that $\mathrm{cl}_{M(\mathcal{C})}(\{x, y\})$ covers $\mathrm{cl}_{M(\mathcal{C})}(\{x\})$.

The modular element and the modular pair are core concepts in lattice. As we know, if $a$ is a modular element of $\mathcal{L}$, then $(a, b)$ is a modular pair of $\mathcal{L}$ for all $b \in \mathcal{L}$, which roots in an important result of lattices. The following theorem shows the relationship among modular element, modular pair and rank function of a matroid in lattice $\mathcal{L}(M)$. 
Theorem 3.13. Let $M$ be a matroid and $\mathcal{L}(M)$ the set of all closed sets of $M$.

(1) For all $X, Y \in \mathcal{L}(M),(X, Y)$ is a modular pair of $\mathcal{L}(M)$ if and only if $r_{M}(X \cup Y)+r_{M}(X \cap$ $Y)=r_{M}(X)+r_{M}(Y)$

(2) For all $X \in \mathcal{L}(M), X$ is a modular element of $\mathcal{L}(M)$ if and only if $r_{M}(X \cup Y)+r_{M}(X \cap Y)=$ $r_{M}(X)+r_{M}(Y)$, for all $Y \in \mathcal{L}(M)$.

Proof. (1) According to Lemmas 2.8 and 2.10, we know $(X, Y)$ is a modular pair of $\mathcal{L}(M)$ if and only if $r_{M}(X)+r_{M}(Y)=r_{M}(X \vee Y)+r_{M}(X \wedge Y)=r_{M}\left(c_{M}(X \cup Y)\right)+r_{M}(X \cap Y)=$ $r_{M}(X \cup Y)+r_{M}(X \cap Y)$.

(2) It comes from the definition of modular element and (1).

Let $\left\{A_{i}: i \in \Gamma\right\}$ be the set of atoms of lattice $\mathcal{L}(M(\mathcal{C}))$, where $\Gamma$ denotes the index set. The following theorem shows the relationship among atoms, modular pairs, and modular elements of the lattice.

Theorem 3.14. Let $\mathcal{C}$ be a covering of $E$. For all $i, j \in \Gamma$.

(1) $\left(A_{i}, A_{j}\right)$ is a modular pair of $\mathcal{L}(M(\mathcal{C}))$.

(2) $A_{i}$ is a modular element of $\mathcal{L}(M(\mathcal{C}))$.

Proof. (1) Since $\mathcal{C}$ is a covering, $\mathrm{cl}_{M(\mathcal{C})}(\emptyset)=\emptyset . A_{i}$ and $A_{j}$ are atoms, so $A_{i} \cap A_{j}=\emptyset$. According to Theorem 3.13, we need to prove $r_{M(\mathcal{C})}\left(A_{i} \cup A_{j}\right)+r_{M(\mathcal{C})}\left(A_{i} \cap A_{j}\right)=r_{M(\mathcal{C})}\left(A_{i}\right)+r_{M(\mathcal{C})}\left(A_{j}\right)$, that is, $r_{M(\mathcal{C})}\left(A_{i} \cup A_{j}\right)=2$. According to the submodular inequality of $r_{M(\mathcal{C})}$, we have $r_{M(\mathcal{C})}\left(A_{i} \cup\right.$ $\left.A_{j}\right)+r_{M(\mathcal{C})}\left(A_{i} \cap A_{j}\right) \leq r_{M(\mathcal{C})}\left(A_{i}\right)+r_{M(\mathcal{C})}\left(A_{j}\right)$, that is, $1 \leq r_{M(\mathcal{C})}\left(A_{i} \cup A_{j}\right) \leq 2$. If $r_{M(\mathcal{C})}\left(A_{i} \cup A_{j}\right)=$ $1=r_{M(\mathcal{C})}\left(A_{i}\right)$, then $A_{j} \subseteq \mathrm{cl}_{M(\mathcal{C})}\left(A_{i}\right)=A_{i}$ which contradicts that $A_{i} \cap A_{j}=\emptyset$.

(2) $A_{i}$ is a modular element of $\mathcal{L}(M(\mathcal{C}))$ if and only if $r_{M(\mathcal{C})}\left(A_{i} \cup A\right)+r_{M(\mathcal{C})}\left(A_{i} \cap A\right)=$ $r_{M(\mathcal{C})}\left(A_{i}\right)+r_{M(\mathcal{C})}(A)$ for all $A \in \mathcal{L}(M(\mathcal{C}))$.

Case 1. If $A_{i}$ and $A$ are comparable, that is, $A_{i} \subseteq A$, then $r_{M(\mathcal{C})}\left(A_{i} \cup A\right)+r_{M(\mathcal{C})}\left(A_{i} \cap A\right)=$ $r_{M(\mathcal{C})}\left(A_{i}\right)+r_{M(\mathcal{C})}(A)$.

Case 2. If $A_{i}$ and $A$ are not comparable, there are two cases. One is that $A$ is an atom of $\mathcal{L}(M(\mathcal{C}))$, the other is that $A$ is not an atom of $\mathcal{L}(M(\mathcal{C}))$. If $A$ is an atom of $\mathcal{L}(M(\mathcal{C}))$, then we obtain the result from (1). If $A$ is not an atom of $\mathcal{L}\left(M(\mathcal{C})\right.$ ), then $A \cap A_{i}=\emptyset$. Hence, $r_{M(\mathcal{C})}(A) \leq r_{M(\mathcal{C})}\left(A \cup A_{i}\right) \leq r_{M(\mathcal{C})}(A)+1$. If $r_{M(\mathcal{C})}\left(A \cup A_{i}\right)=r_{M(\mathcal{C})}(A)$, then $A_{i} \subseteq \mathrm{cl}_{M(\mathcal{C})}(A)=A$ which contradicts that $A_{i} \cap A=\emptyset$. Hence, $r_{M(\mathcal{C})}\left(A \cup A_{i}\right)=r_{M(\mathcal{C})}(A)+1$.

In a word, for all $A \in \mathcal{L}(M(\mathcal{C})), r_{M(\mathcal{C})}\left(A_{i} \cup A\right)+r_{M(\mathcal{C})}\left(A_{i} \cap A\right)=r_{M(\mathcal{C})}\left(A_{i}\right)+r_{M(\mathcal{C})}(A)$, that is, $A_{i}$ is a modular element of $\mathcal{L}(M(\mathcal{C}))$ for all $i \in \Gamma$.

When a covering degenerates into a partition, it is not difficult for us to obtain the following result.

Corollary 3.15. Let $D$ be a partition of E. For all $P_{i}, P_{j} \in D$ :

(1) $\left(P_{i}, P_{j}\right)$ is a modular pair of $\mathcal{L}(M(D))$.

(2) $P_{i}$ is a modular element of $\mathcal{L}(M(D))$.

The following lemma shows how to induce a matroid by a lattice. In fact, if a function $f$ on a lattice is nonnegative, integer-valued, submodular and $f(\emptyset)=0$, then it can determine a matroid. 
Lemma 3.16 (see [19]). Let $\mathcal{L}_{E}$ be a lattice of subsets of a set $E$ such that $\mathcal{L}_{E}$ is closed under intersection, and contains $\emptyset$ and E. Suppose that $f$ is a nonnegative, integer-valued, submodular function on $\mathcal{L}_{E}$ for which $f(\emptyset)=0$. Let $\supset\left(\mathcal{L}_{E}, f\right)=\left\{X \subseteq E: f(X) \geq|X \cap T|\right.$, for all $\left.T \in \mathcal{L}_{E}\right\}$. $\supset\left(\mathcal{L}_{E}, f\right)$ is the collection of independent sets of a matroid on $E$.

According to the definition of $\mathcal{L}(M(\mathcal{C}))$, we find that $\mathcal{L}(M(\mathcal{C}))$ is closed under intersection, and contains $\emptyset$ and $E$. Moreover, the rank function of $M(\mathcal{C})$ is a nonnegative, integer-valued, submodular function on $\mathcal{L}\left(M(\mathcal{C})\right.$ for which $r_{M(\mathcal{C})}(\emptyset)=0$. Similar to Lemma 3.16, we can obtain the following theorem.

Theorem 3.17. Let $\mathcal{C}$ be a covering of $E$. We define $\supset\left(\mathcal{L}(M(\mathcal{C})), r_{M(\mathcal{C})}\right)=\left\{X \subseteq E: r_{M(\mathcal{C})}(Y) \geq\right.$ $|X \cap Y|$, for all $Y \in \mathcal{L}(M(\mathcal{C}))\}$, then $M\left(E, \supset\left(\mathcal{L}\left(M(\mathcal{C}), r_{M(\mathcal{C})}\right)\right)\right.$ is a matroid.

For any given matroid $M$, we know that for all $X \subseteq E, X$ is an independent set of $M$ if and only if $r_{M}(X)=|X|$. Moreover, based on the properties of rank function, we have $r_{M}(X) \leq|X|$. Hence, $X$ is an independent set of $M$ if and only if $r_{M}(X) \geq|X|$ for all $X \subseteq E$.

Lemma 3.18. Let $M$ be a matroid. $X$ is an independent set of $M$ if and only if for all closed set $Y$ of $M, r_{M}(Y) \geq|X \cap Y|$.

Proof. " $\Rightarrow$ ": Since for all closed set $Y, X \cap Y \subseteq X$ and $X$ is an independent set, $X \cap Y$ is an independent set of $M$ according to the independent set axiom of a matroid. Hence, we have $r_{M}(Y) \geq r_{M}(X \cap Y) \geq|X \cap Y|$.

“ $\Leftarrow$ ": For all closed set $Y, r_{M}(Y) \geq|X \cap Y|$. Especially, for $Y=\mathrm{cl}_{M}(X)$, we have $r_{M}(X)=$ $r_{M}\left(\mathrm{cl}_{M}(X)\right)=r_{M}(Y) \geq|X \cap Y|=|X|$. Hence, $X$ is an independent set of matroid $M$.

What is the relation between the two matroids induced by a covering and a geometric lattice, respectively? In order to establish the relation between them, we first denote $r_{\mathcal{L}(M(\mathcal{C}))}$ as the rank function of $M\left(E, \supset\left(\mathcal{L}\left(M(\mathcal{C}), r_{M(\mathcal{C})}\right)\right)\right.$ on $E$. The following theorem shows there is a one-to-one correspondence between geometric lattices and transversal matroids in the context of covering-based rough sets.

Theorem 3.19. Let $\mathcal{C}$ be a covering of E. For all $X \subseteq E, \supset\left(\mathcal{L}\left(M(\mathcal{C}), r_{M(\mathcal{C})}\right)=\supset(M(\mathcal{C}))\right.$ and $r_{\mathcal{L}(M(\mathcal{C}))}(X)=r_{M(\mathcal{C})}(X)$.

Proof. According to Lemma 3.18, we know that $\supset\left(\mathcal{L}\left(M(\mathcal{C}), r_{M(\mathcal{C})}\right)=\left\{X \subseteq E: r_{M(\mathcal{C})}(Y) \geq\right.\right.$ $|X \cap Y|$, for all $Y \in \mathcal{L}(M(\mathcal{C}))\}$ and $\supset(\mathcal{C})=\left\{X \subseteq E: r_{M(\mathcal{C})}(X)=|X|\right\}$ are equivalent, that is, $M\left(E, \supset\left(\mathcal{L}\left(M(\mathcal{C}), r_{M(\mathcal{C})}\right)\right)\right.$ and $M(E, \supset(\mathcal{C}))$ are equivalent. So does $r_{\mathcal{L}(M(\mathcal{C}))}(X)=r_{M(\mathcal{C})}(X)$ for all $X \subseteq E$.

When a covering degrades into a partition, we can obtain a matroid $M(P)=(E, \supset(P))$, where $\mathcal{O}(D)=\left\{X \subseteq E\right.$ : for all $\left.P_{i} \in D,\left|X \cap P_{i}\right| \leq 1\right\}$ and $r_{M(D)}(X)=\max \{|I|: I \subseteq X, I \in \supset(D)\}=$ $\left|\left\{P_{i} \in D: P_{i} \cap X \neq \emptyset\right\}\right|$ for all $X \subseteq E$. As we know, for all $X, Y \in \mathcal{L}(M), X \vee Y=\mathrm{cl}_{M}(X \cup Y)$. If the matroid is $M(D)$, then $X \vee Y=X \cup Y$.

Lemma 3.20 (see [25]). If $D$ is a partition of $E$ and $M$ is the matroid, then $c l_{M}=R^{*}$ for all $X \subseteq E$.

Lemma 3.21. Let $D$ be a partition of E. For all $X, Y \in \mathcal{L}(M(D)), X \vee Y=X \cup Y$. 
Proof. $X \vee Y=\mathrm{cl}_{M(p)}(X \cup Y)=R^{*}(X \cup Y)=R^{*}(X) \cup R^{*}(Y)=\mathrm{cl}_{M(p)}(X) \cup \mathrm{cl}_{M(p)}(Y)=X \cup Y$.

Based on the above two lemmas, we can obtain the following proposition.

Proposition 3.22. Let $D$ be a partition of E. For all $X \subseteq E, \supset\left(\mathcal{L}(M(D)), r_{M(p)}\right)=\supset(M(D))$ and $r_{\mathcal{L}(M(p))}(X)=r_{M(p)}(X)$.

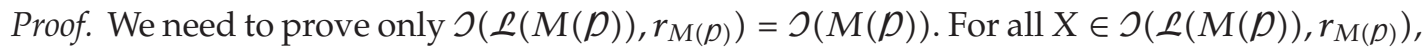
then $r_{M(p)}(Y) \geq|X \cap Y|$ for all $Y \in \mathcal{L}(M(D))$. Since $P_{i} \in \mathcal{L}(M(D)),\left|X \cap P_{i}\right| \leq r_{M(\mathcal{})}\left(P_{i}\right)=1$. Thus $X \in \supset(M(D))$. Hence, $\supset\left(\mathcal{L}\left(M(D), r_{M(D)}\right) \subseteq \supset(M(D))\right.$. According to Lemmas 2.9 and 3.21, for all $Y \in \mathcal{L}(M(D))$, there exists $K \subseteq\{1,2, \ldots, m\}$ such that $Y=\bigvee_{i \in K} P_{i}=\bigcup_{i \in K} P_{i}$. Thus $r_{M(p)}(Y)=|K|$ and $|X \cap Y|=\left|X \cap\left(\bigcup_{i \in K} P_{i}\right)\right|=\left|\bigcup_{i \in K}\left(X \cap P_{i}\right)\right|=\sum_{i \in K}\left|X \cap P_{i}\right|$. For all $X \in \supset(M(D)),\left|X \cap P_{i}\right| \leq 1$. Hence, $|X \cap Y|=\sum_{i \in K}\left|X \cap P_{i}\right| \leq|K|=r_{M(\mathcal{D})}(Y)$, that is, $\supset(M(D)) \subseteq \supset\left(\mathcal{L}(M(D)), r_{M(p)}\right)$.

\section{Two Geometric Lattice Structures of Covering-Based Rough Sets through Approximation Operators}

A geometric lattice structure of covering-based rough sets is established through the transversal matroid induced by a covering, and its characteristics including atoms, modular elements, and modular pairs are studied in Section 3. In this section, we study matroidal structures and the geometric lattice structures from the viewpoint of covering upper approximation operators. The conditions of two types of upper approximation operators to be matroidal closure operators are obtained, and the properties of the matroids and their geometric lattice structures induced by the operators are also established.

Pomykała first studied the second type of covering rough set model [30]. Zhu and Wang studied the axiomatization of this type of upper approximation operator and the relationship between it and the Kuratowski closure operator in [27]. First, we give some properties of this operator.

Proposition 4.1. Let $\mathcal{C}$ be a covering of $E$. SH has the following properties:

(1) $S H(\emptyset)=\emptyset$,

(2) $X \subseteq S H(X)$ for all $X \subseteq E$,

(3) $S H(X \cup Y)=S H(X) \cup S H(Y)$ for all $X, Y \subseteq E$,

(4) $x \in S H(\{y\}) \Leftrightarrow y \in S H(\{x\})$ for all $x, y \in E$,

(5) $X \subseteq Y \subseteq E \Rightarrow S H(X) \subseteq S H(Y)$,

(6) for all $x, y \in E, y \in S H(X \cup\{x\})-S H(X)$, then $x \in S H(X \cup\{y\})$.

Proof. (1)-(5) were proven in $[30,32,33]$. Here we prove only (6). According to (3), we know $\mathrm{SH}(X \cup\{x\})-\mathrm{SH}(X)=\mathrm{SH}(X) \cup \mathrm{SH}(\{x\})-\mathrm{SH}(X)=\mathrm{SH}(\{x\})-\mathrm{SH}(X)$. If $y \in \mathrm{SH}(X \cup\{x\})-$ $\mathrm{SH}(X)$, then $y \in \mathrm{SH}(\{x\})$. According to (4) and (5), we have $x \in \mathrm{SH}(\{y\}) \subseteq \mathrm{SH}(X \cup\{y\})$.

We find that the idempotent of $\mathrm{SH}$ is not valid, so what is the condition that guarantees it holds for $\mathrm{SH}$ ? We have the following conclusion.

Proposition 4.2. Let $\mathcal{C}$ be a covering of $E$. For all $X \subseteq E, S H(S H(X))=S H(X)$ if and only if $\{I(x): x \in E\}$ induced by $\mathcal{C}$ forms a partition of $E$. 
Proof. " $\Leftarrow$ ": According to (2), (5) of Proposition 4.1, we have $\mathrm{SH}(X) \subseteq \mathrm{SH}(\mathrm{SH}(X))$. Now we prove $\mathrm{SH}(\mathrm{SH}(X)) \subseteq \mathrm{SH}(X)$. For all $x \in \mathrm{SH}(\mathrm{SH}(X))$, there exists $y \in \mathrm{SH}(X)$ such that $x \in I(y)$. Since $y \in S H(X)$, there exists $z \in X$ such that $y \in I(z)$. According to the definition of $I(y)$, we know $y \in I(y)$, thus $I(z) \cap I(y) \neq \emptyset$. For $\{I(x): x \in E\}$ forms a partition, $I(z)=I(y)$. Since $x \in I(y), x \in I(z)$, that is, $x \in \mathrm{SH}(X)$, thus $\mathrm{SH}(\mathrm{SH}(X)) \subseteq \mathrm{SH}(X)$.

" $\Rightarrow$ ": In order to prove $\{I(x): x \in E\}$ forms a partition, we need to prove that for all $x, y \in E$, if $I(x) \cap I(y) \neq \emptyset$, then $I(x)=I(y)$. If $I(x) \cap I(y) \neq \emptyset$, then there exists $z \in I(x) \cap$ $I(y)$. For $\operatorname{SH}(\mathrm{SH}(\{x\}))=\cup\{I(u): u \in I(x)\}$ and $z \in I(x)$, then $I(z) \subseteq \operatorname{SH}(\mathrm{SH}(\{x\}))=$ $\mathrm{SH}(\{x\})=I(x)$. Based on the definition of $I(z)$ and $z \in I(x)$, we have $x \in I(z)$, thus $I(x) \subseteq$ $\mathrm{SH}(\mathrm{SH}(\{z\}))=\mathrm{SH}(\{z\})=I(z)$. Hence, $I(x)=I(z)$. Similarly, we can obtain $I(y)=I(z)$, thus $I(x)=I(z)=I(y)$.

The following theorem establishes a necessary and sufficient condition for $\mathrm{SH}$ to be a closure operator.

Theorem 4.3. Let $\mathcal{C}$ be a covering of $E$. SH is a closure operator of a matroid if and only if $\{I(x): x \in$ $E\}$ induced by $C$ forms a partition of $E$.

Proof. It comes from Propositions 4.1 and 4.2 and (2), (5), and (6) of Proposition 2.3.

For a given covering $C$ of $E$, we may as well suppose the set of indiscernible neighborhoods of $E$ as $\{I(x): x \in E\}=\left\{I\left(x_{1}\right), I\left(x_{2}\right), \ldots, I\left(x_{s}\right)\right\}$ where $x_{1}, x_{2}, \ldots, x_{s} \in E$.

Definition 4.4. Let $\mathcal{C}$ be a covering of $E$. We define $\mathcal{I}^{\prime}=\left\{I \subseteq E:\left|I \cap I\left(x_{i}\right)\right| \leq 1\right.$, for all $i \in$ $\{1,2, \ldots, s\}\}$.

As we know, if $\{I(x): x \in E\}=\left\{I\left(x_{1}\right), I\left(x_{2}\right), \ldots, I\left(x_{s}\right)\right\}$ forms a partition of $E$, then $M\left(E, \supset^{\prime}\right)$ is a matroid and $\mathrm{SH}$ is the closure operator of a matroid. Thus SH can determine a matroid, and the independent sets of the matroid induced by it are established as follows:

$$
\supset_{\mathrm{SH}}(\mathcal{C})=\{I \subseteq E: \forall x \in I, x \notin \mathrm{SH}(I-\{x\})\} .
$$

The following proposition shows $M\left(E, \supset_{\mathrm{SH}}\right)=M\left(E, \supset^{\prime}\right)$ under the condition that $\{I(x): x \in$ $E$ \} forms a partition of $E$.

Proposition 4.5. Let $\mathcal{C}$ be a covering of $E$. If $\{I(x): x \in E\}$ induced by $\mathcal{C}$ forms a partition of $E$, then $M\left(E, \supset_{S H}(\mathcal{C})\right)$ is a matroid and $\supset_{S H}(\mathcal{C})=\supset^{\prime}$.

Proof. Let $\supset_{\mathrm{cl}}=\{I \subseteq E$ : for all $x \in I, x \notin \mathrm{cl}(I-\{x\})\}$. we know that if an operator $\mathrm{cl}$ satisfies (1)-(4) of Proposition 2.3, $M\left(E, \supset_{\mathrm{cl}}\right)$ is a matroid. $\{I(x): x \in U\}$ induced by $C$ forms a partition, hence, $M\left(E, \supset_{\mathrm{SH}}(\mathcal{C})\right)$ is a matroid. Since $\mathrm{SH}(I)=\bigcup_{y \in I} I(y), \operatorname{SH}(I-\{x\})=$ $\bigcup_{y \in I-\{x\}} I(y)$. According to the definition of $I(x)$, we know $x \in I(x)$. On one hand, for all $I \in \supset_{\mathrm{SH}}(\mathcal{C})$, we know that for all $x \in I, x \notin \mathrm{SH}(I-\{x\})$, that is, for all $y \in I$ and $y \neq x, x \notin I(y)$. If $I \notin \supset^{\prime}$, that is, there exists $1 \leq i \leq s$ such that $\left|I \cap I\left(x_{i}\right)\right| \geq 2$, then we may as well suppose there exist $u, v$ such that $u, v \in I\left(x_{i}\right)$ and $u, v \in I$. Since $u \in I(u), v \in I(v)$ and $\{I(x): x \in E\}$ forms a partition, $I(u)=I(v)=I\left(x_{i}\right)$. Based on that, we know there exists $u \in I$ and $u \neq v$ such that $u \in I(v)$, that implies contradiction. Hence, $I \in \supset^{\prime}$, that is, $\supset_{\mathrm{SH}}(\mathcal{C}) \subseteq \supset^{\prime}$. On the other hand, if $I \notin \supset_{\mathrm{SH}}(\mathcal{C})$, then there exists $x \in I$ such that $x \in \mathrm{SH}(I-\{x\})=\bigcup_{y \in I-\{x\}} I(y)$. That implies that there exists $y \in I$ and $y \neq x$ such that $x \in I(y)$. Since $x \in I(x)$ and $\{I(x): x \in U\}$ 
forms a partition, $I(x)=I(y)$. Thus $x, y \in I \cap I(x)$, that implies $|I \cap I(x)| \geq 2$, that is, $I \notin \supset^{\prime}$. Hence, $\supset^{\prime} \subseteq \supset_{\mathrm{SH}}(\mathcal{C})$.

We denote the rank function of $M\left(E, \supset_{\mathrm{SH}}(\mathcal{C})\right)$ by $r_{\mathrm{SH}}$. Then some properties of $M\left(E, \supset_{\mathrm{SH}}(\mathcal{C})\right)$ are established in the following proposition.

Proposition 4.6. Let $\mathcal{C}$ be a covering of $E$. If $\{I(x): x \in E\}=\left\{I\left(x_{1}\right), I\left(x_{2}\right), \ldots, I\left(x_{s}\right)\right\}$ induced by $C$ forms a partition of $E$, then

(1) $X$ is a base of $M\left(E, \supset_{S H}(\mathcal{C})\right)$ if and only if $\left|X \cap I\left(x_{i}\right)\right|=1$ for all $i \in\{1,2, \ldots, s\}$. Moreover, $M\left(E, \supset_{S H}(\mathcal{C})\right)$ has $\left|I\left(x_{1}\right)\right|\left|I\left(x_{2}\right)\right| \cdots\left|I\left(x_{s}\right)\right|$ bases.

(2) For all $X \subseteq E, r_{S H}(X)=\left|\left\{I\left(x_{i}\right): I\left(x_{i}\right) \cap X \neq \emptyset, i=1,2, \ldots, s\right\}\right|$.

(3) $X$ is a dependent set of $M\left(E, \supset_{S H}(\mathcal{C})\right)$ if and only if there exists $I\left(x_{i}\right) \in\{I(x): x \in E\}$ such that $\left|I\left(x_{i}\right) \cap X\right|>1$.

(4) $X$ is a circuit of $M\left(E, \bigcirc_{S H}(\mathcal{C})\right)$ if and only if there exists $I\left(x_{i}\right) \in\{I(x): x \in E\}$ such that $X \subseteq I\left(x_{i}\right)$ and $|X|=2$.

Proof. (1) According to the definition of base of a matroid, we know that $X$ is a base of $M\left(E, \supset_{\mathrm{SH}}(\mathcal{C})\right) \Leftrightarrow X$ is a maximal independent set of $M\left(E, \supset_{\mathrm{SH}}(\mathcal{C})\right) \Leftrightarrow\left|X \cap I\left(x_{i}\right)\right|=1$ for all $i \in$ $\{1,2, \ldots, s\}$ because $\supset_{\mathrm{SH}}(\mathcal{C})=\supset^{\prime}$. Since $X$ is a base of $M\left(E, \supset_{\mathrm{SH}}(\mathcal{C})\right)$ and $I\left(x_{1}\right), I\left(x_{2}\right), \ldots, I\left(x_{s}\right)$ are different, $M\left(E, \supset_{\mathrm{SH}}(\mathcal{C})\right)$ has $\left|I\left(x_{1}\right)\right|\left|I\left(x_{2}\right)\right| \cdots\left|I\left(x_{s}\right)\right|$ bases.

(2) According to the definition of rank function, we know $r_{\mathrm{SH}}(X)=\left|B_{X}\right|=\mid\left\{I\left(x_{i}\right)\right.$ : $\left.\left|B_{X} \cap I\left(x_{i}\right)\right|=1\right\}|\leq|\left\{I\left(x_{i}\right): X \cap I\left(x_{i}\right) \neq \emptyset\right\} \mid$, where $B_{X}$ is a maximal independent set included in $X$. Now we just need to prove the inequality $\left|\left\{I\left(x_{i}\right):\left|B_{X} \cap I\left(x_{i}\right)\right|=1\right\}\right|<\left|\left\{I\left(x_{i}\right): X \cap I\left(x_{i}\right) \neq \emptyset\right\}\right|$ does not hold; otherwise, there exists $1 \leq i \leq s$ such that $I\left(x_{i}\right) \cap X \neq \emptyset$ and $I\left(x_{i}\right) \cap B_{X}=\emptyset$ because $B_{\mathrm{X}} \in \supset_{\mathrm{SH}}(\mathcal{C})$. Thus there exists $e_{i} \in I\left(x_{i}\right) \cap X$ such that $B_{\mathrm{X}} \cup\left\{e_{i}\right\} \subseteq X$ and $B_{\mathrm{X}} \cup\left\{e_{i}\right\} \in \supset_{\mathrm{SH}}(\mathcal{C})$. That contradicts the assumption that $B_{X}$ is a maximal independent set included in $X$. Hence, $r_{\mathrm{SH}}(X)=\left|\left\{I\left(x_{i}\right): I\left(x_{i}\right) \cap X \neq \emptyset, i=1,2, \ldots, s\right\}\right|$.

(3) According to the definition of dependent set, we know that $X$ is a dependent set $\Leftrightarrow X \notin \supset_{\mathrm{SH}}(\mathcal{C}) \Leftrightarrow$ there exists $i \in\{1,2, \ldots, s\}$ such that $\left|X \cap I\left(x_{i}\right)\right|>1$.

(4) " $\Rightarrow$ ": As we know, a circuit is a minimal dependent set. $X$ is a circuit of $M\left(E, \supset_{\mathrm{SH}}(\mathcal{C})\right)$, then there exists $i \in\{1,2, \ldots, s\}$ such that $\left|I\left(x_{i}\right) \cap X\right|=2$. Now we just need to prove $|X|=2$; otherwise, we may as well suppose $X=\{x, y, z\}$ where $x, y \in I\left(x_{i}\right) \cap X$. Thus we can obtain $\left|(X-\{z\}) \cap I\left(x_{i}\right)\right|=2$, that is, $X-\{z\} \notin \supset_{\mathrm{SH}}(\mathcal{C})$. That contradicts the minimality of circuit. Combining $|X|=2$ with $\left|I\left(x_{i}\right) \cap X\right|=2$, we have $X \subseteq I\left(x_{i}\right)$.

" $\Leftarrow$ ": Since $|X|=2$, we may as well suppose $X=\{x, y\}$, and $\left|X \cap I\left(x_{i}\right)\right|=2$ because there exists $I\left(x_{i}\right) \in\{I(x): x \in E\}$ such that $X \subseteq I\left(x_{i}\right)$, thus $X$ is a dependent set. For all $j \in$ $\{1,2, \ldots, s\},\left|\{x\} \cap I\left(x_{j}\right)\right| \leq 1$ and $\left|\{y\} \cap I\left(x_{j}\right)\right| \leq 1$ which implies $\{x\}$ and $\{y\}$ are independent sets, hence $X$ is a circuit of $M\left(E, \supset_{\mathrm{SH}}(\mathcal{C})\right)$.

For a covering $\mathcal{C}$ of $E$, we denote $\mathcal{L}_{\mathrm{SH}}(M(\mathcal{C}))$ as the set of all closed sets of $M\left(E, \supset_{\mathrm{SH}}(\mathcal{C})\right)$. When $\{I(x): x \in E\}=\left\{I\left(x_{1}\right), I\left(x_{2}\right), \ldots, I\left(x_{S}\right)\right\}$ forms a partition of $E$, then for all $X, Y \in \mathcal{L}_{\mathrm{SH}}(M(\mathcal{C})), X \wedge Y=X \cap Y, X \vee Y=\mathrm{SH}(X \cup Y)=\mathrm{SH}(X) \cup \mathrm{SH}(Y)=X \cup Y$ and $\mathrm{SH}(\emptyset)=\emptyset$. 
Proposition 4.7. Let $\mathcal{C}$ be a covering of $E$. If $\{I(x): x \in E\}=\left\{I\left(x_{1}\right), I\left(x_{2}\right), \ldots, I\left(x_{s}\right)\right\}$ forms a partition of $E$, then

(1) $I\left(x_{1}\right), I\left(x_{2}\right), \ldots, I\left(x_{s}\right)$ are all atoms of $\mathcal{L}\left(M_{S H}\right)$.

(2) For all $x, y \in E$ and $x \neq y$, there does not exist $I(z) \in\left\{I\left(x_{1}\right), I\left(x_{2}\right), \ldots, I\left(x_{s}\right)\right\}$ such that $x, y \in I(z)$ if and only if $\operatorname{SH}(\{x, y\})$ covers $\operatorname{SH}(\{x\})$ or $\operatorname{SH}(\{y\})$.

(3) For all $i, j \in\{1,2, \ldots, s\},\left(I\left(x_{i}\right), I\left(x_{j}\right)\right)$ is a modular pair of $\mathcal{L}_{S H}(M(\mathcal{C}))$.

(4) For all $i \in\{1,2, \ldots, s\}, I\left(x_{i}\right)$ is a modular element of $\mathcal{L}_{S H}(M(\mathcal{C}))$.

Proof. (1) comes from Corollary 3.10, Theorem 4.3 and Proposition 4.5. Based on Proposition 3.12 and Theorem 4.3, we can obtain (2). According to Corollary 3.15, Theorem 4.3 and Proposition 4.5, it is easy to obtain (3) and (4).

Based on Theorem 4.3, we know that a necessary and sufficient condition for $\mathrm{SH}$ to be a closure operator of a matroid is that $\{I(x): x \in E\}$ forms a partition of $E$. The following two propositions show what kind of coverings can satisfy that condition.

Lemma 4.8. Let $\mathcal{C}$ be a covering of $E$ and $K \in \mathcal{C}$. If $K$ is an immured element, then $I(x)$ is the same in $C$ as in $C-\{K\}$.

Proof. If $x \notin K$, then $I_{\mathcal{C}}(x)=I_{\mathcal{C}-\{K\}}(x)$. If $x \in K$, then $I_{\mathcal{C}}(x)=\bigcup_{x \in K^{\prime}} K^{\prime} \cup K$. Since $K$ is an immured element, there exists $x \in K^{\prime}$ such that $K \subseteq K^{\prime}$. Thus $I_{\mathcal{C}}(x)=\bigcup_{x \in K^{\prime}} K^{\prime} \cup K=$ $\bigcup_{x \in K^{\prime}} K^{\prime}=I_{\mathcal{C}-\{K\}}(x)$. Hence, $I(x)$ is the same in $\mathcal{C}$ as in $\mathcal{C}-\{K\}$.

Proposition 4.9. Let $\mathcal{C}$ be a covering of $E$. If exclusion $(\mathcal{C})$ is a partition of $E$, then $\{I(x): x \in E\}$ induced by $C$ also forms a partition of $E$.

Proof. Since exclusion $(\mathcal{C})$ is a partition of $E,\{I(x): x \in E\}$ induced by exclusion $(\mathcal{C})$ forms a partition. Suppose $\left\{K_{1}, K_{2}, \ldots, K_{s}\right\}$ is the set of all immured elements of $\mathcal{C}$. According to Lemma 4.8, we know for all $x \in E, I(x)$ is the same in exclusion $(\mathcal{C})$ as in exclusion $(\mathcal{C}) \cup\left\{K_{1}\right\}$. Thus $\{I(x): x \in E\}$ induced by exclusion $(\mathcal{C}) \cup\left\{K_{1}\right\}$ forms a partition of $E$. And the rest may be deduced by analogy, we know that for all $x \in E, I(x)$ is the same in $\operatorname{exclusion}(\mathcal{C})$ as in $\mathcal{C}$, thus $\{I(x): x \in E\}$ induced by $\mathcal{C}$ forms a partition of $E$.

The proposition below establishes a necessary and sufficient condition for $\{I(x): x \in$ $E$ \} forms a partition of $E$ from the viewpoint of coverings.

Proposition 4.10. Let $\mathcal{C}$ be a covering of $E .\{I(x): x \in E\}$ induced by $\mathcal{C}$ forms a partition of $E$ if and only if $\mathcal{C}$ satisfies (TRA) condition: For all $x, y, z \in E, x, z \in K_{1} \in \mathcal{C}, y, z \in K_{2} \in \mathcal{C}$, there exists $K_{3} \in \mathcal{C}$ such that $x, y \in K_{3}$.

Proof. " $\Leftarrow "$ : For all $x, y \in E, I(x) \cap I(y)=\emptyset$ or $I(x) \cap I(y) \neq \emptyset$. If $I(x) \cap I(y) \neq \emptyset$, then there exists $z \in I(x)$ and $z \in I(y)$. According to the definition of $I(x)$ and $I(y)$, there exist $K_{1}, K_{2}$ such that $x, z \in K_{1}$ and $y, z \in K_{2}$. According to the hypothesis, we know there exists $K_{3} \in \mathcal{C}$ such that $x, y \in K_{3}$. Now we need to prove only $I(x)=I(y)$. For all $u \in I(x)$, there exists $K \in \mathcal{C}$ such that $u, x \in K$. Since $x, y \in K_{3}$, there exists $K^{\prime} \in \mathcal{C}$ such that $u, y \in K^{\prime}$, that is, $u \in I(y)$, thus $I(x) \subseteq I(y)$. Similarly, we can prove $I(y) \subseteq I(x)$. Hence, $I(x)=I(y)$, that is, $\{I(x): x \in E\}$ forms a partition of $E$. 
" $\Rightarrow$ ": For all $x, y, z \in E, x, z \in K_{1} \in \mathcal{C}$ and $y, z \in K_{2} \in \mathcal{C}$, we can obtain $z \in I(x)$ and $z \in I(y)$. That implies $I(x) \cap I(y) \neq \emptyset$. Since $\{I(x): x \in E\}$ forms a partition of $E, I(x)=I(y)$. Thus there exists $K_{3} \in \mathcal{C}$ such that $x, y \in K_{3}$.

The following theorem presents a necessary and sufficient condition for $\mathrm{SH}$ to be a closure operator of a matroid from the viewpoint of coverings.

Theorem 4.11. Let $\mathcal{C}$ be a covering of $E$. $\mathcal{C}$ satisfies (TRA) condition if and only if $S H$ induced by $C$ is a closure operator of a matroid.

Proof. It comes from Theorem 4.3 and Proposition 4.10 .

The sixth type of covering-based upper approximation operator was first defined in [31]. Xu and Wang introduced this type of covering-based rough set model and studied the relationship between it and binary relation based rough set model. The following proposition gives some properties of this covering upper approximation operator.

Proposition 4.12 (see [26]). Let $\mathcal{C}$ be a covering of $E$. XH has the following properties:

(1) $X H(E)=E$,

(2) $X H(\emptyset)=\emptyset$,

(3) $X \subseteq X H(X)$ for all $X \subseteq E$,

(4) $X H(X \cup Y)=X H(X) \cup X H(Y)$ for all $X, Y \subseteq E$,

(5) $X H(X H(X))=X H(X)$ for all $X \subseteq E$,

(6) for all $X \subseteq Y \subseteq E \Rightarrow X H(X) \subseteq X H(Y)$.

From the above proposition, we find that $\mathrm{XH}$ does not satisfy the (4) of Proposition 2.3. The following proposition establishes a necessary and sufficient condition for $\mathrm{XH}$ to satisfy the condition.

Proposition 4.13. Let $\mathcal{C}$ be a covering of $E$. For all $x, y \in E$ and $X \subseteq E, X H$ satisfies

$$
y \in X H(X \bigcup\{x\})-X H(X) \Longrightarrow x \in X H(X \bigcup\{y\})
$$

if and only if $\{N(x): x \in E\}$ forms a partition of $E$.

Proof. $\Rightarrow$ : For all $x, y \in E$, if $N(x) \cap N(y) \neq \emptyset$, then there exists $z \in N(x) \cap N(y)$. Let $X=\emptyset$. According to (2) of Proposition 4.12, we know that if $y \in \mathrm{XH}(\{x\})$ then $x \in \mathrm{XH}(y)$, that is, if $x \in N(y)$ then $y \in N(x)$. Since $z \in N(x), N(z) \subseteq N(x)$. According to the assumption, we also have $x \in N(z)$, that is, $N(x) \subseteq N(z)$. Thus $N(x)=N(z)$. Similarly, $z \in N(y)$, we have $N(z)=N(y)$. Thus $N(x)=N(z)=N(y)$. Hence, $\{N(x): x \in E\}$ forms a partition of $E$. $\Leftarrow$ : Since $\mathrm{XH}(X \cup Y)=\mathrm{XH}(X) \cup \mathrm{XH}(Y)$ for all $X, Y \subseteq U, y \in \mathrm{XH}(X \cup\{x\})-\mathrm{XH}(X)=$ $\mathrm{XH}(X) \cup \mathrm{XH}(\{x\})-\mathrm{XH}(X)=\mathrm{XH}(\{x\})-\mathrm{XH}(X)$. Now we prove $x \in \mathrm{XH}(\{y\})$. Since $y \in$ $\mathrm{XH}(\{x\}), x \in N(y)$. Because the fact that $\{N(x): x \in E\}$ forms a partition of $E, x \in N(x)$ and $x \in N(y)$, we have $N(x)=N(y)$, thus $y \in N(x)$, that is, $x \in \mathrm{XH}(\{y\})$. Hence $x \in \mathrm{XH}(\{y\}) \subseteq$ $\mathrm{XH}(\mathrm{X} \cup\{y\})$. 
The following theorem establishes a necessary and sufficient condition for $\mathrm{XH}$ to be a closure operator of a matroid.

Theorem 4.14. Let $\mathcal{C}$ be a covering of $E .\{N(x): x \in E\}$ induced by $\mathcal{C}$ forms a partition of $E$ if and only if XH is a closure operator of a matroid.

Proof. It comes from (3), (5), and (6) of Propositions 4.12 and 4.13.

For convenience, for a given covering $\mathcal{C}$ of $E$, we may as well suppose the set of all neighborhoods as $\{N(x): x \in E\}=\left\{N\left(x_{1}\right), N\left(x_{2}\right), \ldots, N\left(x_{t}\right)\right\}$ where $x_{1}, x_{2}, \ldots, x_{t} \in E$.

Definition 4.15. Let $\mathcal{C}$ be a covering of $E$. We define $\mathcal{J}^{\prime \prime}=\left\{I \subseteq E:\left|I \cap N\left(x_{i}\right)\right| \leq 1\right.$, for all $i \in$ $\{1,2, \ldots, t\}\}$.

Theorem 4.14 indicates that if $\{N(x): x \in E\}$ forms a partition of $E$, then $\mathrm{XH}$ is a closure operator of a matroid. Hence, $\mathrm{XH}$ can determine a matroid, and the independent sets of the matroid induced by it are established as follows:

$$
\supset_{\mathrm{XH}}(\mathcal{C})=\{I \subseteq E: \forall x \in I, x \notin \mathrm{XH}(I-\{x\})\} .
$$

Similar to the case of $\mathrm{SH}$, we can obtain the following results.

Proposition 4.16. Let $\mathcal{C}$ be a covering of $E$. If $\{N(x): x \in E\}$ induced by $\mathcal{C}$ forms a partition of $E$, then $M\left(E, \supset_{X H}(\mathcal{C})\right)$ is a matroid and $\supset_{X H}(\mathcal{C})=\supset^{\prime \prime}$.

$r_{\mathrm{XH}}$ denotes the rank function of $M\left(E, \supset_{\mathrm{XH}}(\mathcal{C})\right)$ and $\mathcal{L}_{\mathrm{XH}}(M(\mathcal{C}))$ denotes the set of all closed sets of $M\left(E, \supset_{\mathrm{XH}}(\mathcal{C})\right)$. Then we can obtain the following proposition.

Proposition 4.17. Let $\mathcal{C}$ be a covering of $E$. If $\{N(x): x \in E\}=\left\{N\left(x_{1}\right), N\left(x_{2}\right), \ldots, N\left(x_{t}\right)\right\}$ induced by $\mathcal{C}$ forms a partition of $E$, then

(1) $X$ is a base of $M\left(E, \supset_{X H}(\mathcal{C})\right)$ if and only if $\left|X \cap N\left(x_{i}\right)\right|=1(1 \leq i \leq t)$, and $M\left(E, \supset_{X H}(\mathcal{C})\right)$ has $\left|N\left(x_{1}\right)\right|\left|N\left(x_{2}\right)\right| \cdots\left|N\left(x_{s}\right)\right|$ bases.

(2) For all $X \subseteq E, r_{X H}(X)=\left|\left\{N\left(x_{i}\right): N\left(x_{i}\right) \cap X \neq \emptyset, 1 \leq i \leq t\right\}\right|$.

(3) $X$ is a circuit of $M\left(E, \supset_{X H}(\mathcal{C})\right)$ if and only if there exists $N\left(x_{i}\right) \in\{N(x): x \in E\}$ such that $X \subseteq N\left(x_{i}\right)$ and $|X|=2$.

(4) $X$ is a dependent set of $M\left(E, \supset_{\mathrm{XH}}(\mathcal{C})\right)$ if and only if there exists $N\left(x_{i}\right) \in\{N(x): x \in E\}$ such that $\left|N\left(x_{i}\right) \cap X\right|>1$.

(5) $\left\{N\left(x_{1}\right), N\left(x_{2}\right), \ldots, N\left(x_{t}\right)\right\}$ is the set of all atoms of lattice $\mathcal{L}_{\mathrm{XH}}(M(\mathcal{C}))$.

(6) For all $x, y \in E$ and $x \neq y$, there does not exist $N(z) \in\left\{N\left(x_{1}\right), N\left(x_{2}\right), \ldots, N\left(x_{t}\right)\right\}$ such that $x, y \in N(z)$ if and only if $\mathrm{XH}(\{x, y\})$ covers $\mathrm{XH}(\{x\})$ or $\mathrm{XH}(\{y\})$.

(7) For all $i, j \in\{1,2, \ldots, t\},\left(N\left(x_{i}\right), N\left(x_{j}\right)\right)$ is a modular pair of lattice $\mathcal{L}_{\mathrm{XH}}(M(\mathcal{C}))$.

(8) For all $i \in\{1,2, \ldots, t\}, N\left(x_{i}\right)$ is a modular element of lattice $\mathcal{L}_{\mathrm{XH}}(M(\mathcal{C}))$.

The proof of Propositions 4.16 and 4.17 is similar to that of Propositions 4.5, 4.6, and 4.7 , respectively. So we omit the proofs of them. Similar to the case of SH, we also study what kind of coverings can make $\{N(x): x \in E\}$ form a partition of $E$. This paper establishes 
only two kinds of coverings. There are some coverings which satisfy the condition appear in $[34,35]$.

Lemma 4.18. Let $C$ be a covering on $E$ and $K$ be reducible in $C$. For all $x \in U, N(x)$ is the same in $C$ as in $C-\{K\}$.

Proof. For all $x \in E, M d(x)$ is the same for covering $\mathcal{C}$ and covering $\mathcal{C}-\{K\}$, so $N(x)=$ $\cap M d(x)$ is the same for the covering $\mathcal{C}$ and covering $\mathcal{C}-\{K\}$.

Proposition 4.19. Let $\mathcal{C}$ be a covering of $E$. If reduct $(\mathcal{C})$ is a partition of $E$, then $\{N(x): x \in E\}$ induced by $C$ is also a partition of $E$.

Proof. Since reduct $(\mathcal{C})$ is a partition of $E,\{N(x): x \in E\}$ induced by reduct $(\mathcal{C})$ forms a partition of $E$. Suppose $\left\{K_{1}, K_{2}, \ldots, K_{s}\right\}$ is the set all reducible elements of $\mathcal{C}$. According to Lemma 4.18, we know that for all $x \in E, N(x)$ is the same in $\operatorname{reduct}(\mathcal{C})$ as in $\operatorname{reduct}(\mathcal{C}) \cup\left\{K_{1}\right\}$, thus $\{N(x): x \in E\}$ induced by reduct $(\mathcal{C}) \cup\left\{K_{1}\right\}$ forms a partition of $E$. And the rest may be deduced by analogy, then we can obtain for all $x \in E, N(x)$ is the same in reduct $(\mathcal{C})$ as in $\mathcal{C}$, thus $\{N(x): x \in E\}$ induced by $\mathcal{C}$ forms a partition of $E$.

The following proposition establishes a sufficient condition for $\{N(x): x \in E\}$ to be a partition of $E$ from the viewpoint of coverings.

Proposition 4.20. Let $\mathcal{C}$ be a covering of $E$. If $\mathcal{C}$ satisfies (EQU) condition: For all $K \in \mathcal{C}$, for all $x, y \in K$, the number of blocks which contain $x$ is equal to that of blocks which contain $y$, then $\{N(x): x \in E\}$ induced by $\mathcal{C}$ forms a partition of $E$.

Proof. For all $x, y \in E$, if $N(x) \cap N(y) \neq \emptyset$, then there exists $z \in E$ such that $z \in N(x)$ and $z \in$ $N(y)$, that is, the blocks which contain $x$ also contain $z$ and the blocks which contain $y$ also contain $z$. Hence, there exist $K_{i}, K_{i}^{\prime}$ such that $x, z \in K_{i}$ and $y, z \in K_{i}^{\prime}$. Without loss of generality, we suppose $\left\{K_{1}, K_{2}, \ldots, K_{s}\right\}$ is the set of all blocks which contain $x$ and $\left\{K_{1}^{\prime}, K_{2}^{\prime}, \ldots, K_{t}^{\prime}\right\}$ is the set of all blocks which contain $y$. Since the number of blocks which contain $z$ is equal to that of blocks which contain $x,\left\{K_{1}, K_{2}, \ldots, K_{s}\right\}$ is the set of all blocks which contain $z$, thus $\left\{K_{1}^{\prime}, K_{2}^{\prime}, \ldots, K_{t}^{\prime}\right\} \subseteq\left\{K_{1}, K_{2}, \ldots, K_{s}\right\}$. Hence, $N(x) \subseteq N(y)$. Similarly, we can prove $N(y) \subseteq$ $N(x)$. Hence, $N(x)=N(y)$, that is, $\{N(x): x \in E\}$ forms a partition of $E$.

Based on Theorem 4.14, Propositions 4.19 and 4.20, we can obtain the following two corollaries.

Corollary 4.21. Let $\mathcal{C}$ be a covering of $E$. If reduct $(\mathcal{C})$ is a partition of $E$, then $\mathrm{XH}$ is a closure operator of a matroid.

Corollary 4.22. Let $\mathcal{C}$ be a covering on E. If $\mathcal{C}$ satisfies (EQU) condition, then XH is a closure operator of a matroid.

\section{Relationships among Three Geometric Lattice Structures of Covering-Based Rough Sets}

In Section 3, the properties of the geometric lattice induced by a covering have been studied by the matroid $M(E, \supset(\mathcal{C}))$. Section 4 presents three sufficient and necessary conditions for 
two types of covering upper approximation operators to be closure operators of matroids. Moreover, we exhibit two types of matroidal structures through closure axioms, and then obtain two geometric lattice structures of covering-based rough sets. In this section, we study the relationship among above three types of geometric lattices through corresponding matroids. We also discuss the reducible element and the immured element's influence on the relationship among this three types of matroidal structures and geometric lattice structures.

The following proposition shows the relationship between $\supset_{\mathrm{SH}}(\mathcal{C})$ and $\supset(\mathcal{C})$, and the relationship between $\mathcal{L}_{\mathrm{SH}}(M(\mathcal{C}))$ and $\mathcal{L}(M(\mathcal{C}))$.

Proposition 5.1. Let $\mathcal{C}$ be a covering of $E$. If $S H$ induced by $\mathcal{C}$ is a closure operator, then $\supset_{S H}(\mathcal{C}) \subseteq$ $\supset(\mathcal{C})$ and $\mathcal{L}_{S H}(M(\mathcal{C})) \subseteq \mathcal{L}(M(\mathcal{C}))$.

Proof. Since SH induced by $\mathcal{C}$ is a closure operator, $\{I(x): x \in E\}=\left\{I\left(x_{1}\right), I\left(x_{2}\right), \ldots, I\left(x_{s}\right)\right\}$ forms a partition of $E$. For all $I \in \supset_{\mathrm{SH}}(\mathcal{C})$, suppose $I=\left\{i_{1}, i_{2}, \ldots, i_{\alpha}\right\}(\alpha \leq s)$ such that $i_{1} \in I\left(x_{i_{1}}\right), i_{2} \in I\left(x_{i_{2}}\right), \ldots, i_{\alpha} \in I\left(x_{i_{\alpha}}\right)$ and $\left\{I\left(x_{i_{1}}\right), I\left(x_{i_{2}}\right), \ldots, I\left(x_{i_{\alpha}}\right)\right\} \subseteq\left\{I\left(x_{1}\right), I\left(x_{2}\right), \ldots, I\left(x_{s}\right)\right\}$. According to the definition of $I(x)$, there exists $\left\{K_{i_{1}}, K_{i_{2}}, \ldots, K_{i_{\alpha}}\right\} \subseteq \mathcal{C}$ such that $i_{1} \in K_{i_{1}}, i_{2} \in$ $K_{i_{2}}, \ldots, i_{\alpha} \in K_{i_{\alpha}}$. Since $\left\{I\left(x_{1}\right), I\left(x_{2}\right), \ldots, I\left(x_{s}\right)\right\}$ forms a partition of $E$, thus $\left\{K_{i_{1}}, K_{i_{2}}, \ldots, K_{i_{\alpha}}\right\}$ are different blocks. According to the definition of transversal matroid, we have $I \in \supset$. Hence, $\supset_{\mathrm{SH}}(\mathcal{C}) \subseteq \supset(\mathcal{C})$.

For all $X \in \mathcal{L}_{\mathrm{SH}}(M(\mathcal{C})), X=\mathrm{SH}(X)=\bigcup_{x \in X} I(x)$. Now we need to prove $X \in \mathcal{L}(M(\mathcal{C}))$, that is, $\mathrm{cl}_{M(\mathcal{C})}(X)=X=\left\{x \mid r_{M(\mathcal{C})}(X)=r_{M(\mathcal{C})}(X \cup\{x\})\right\}$. Since $X \subseteq \mathrm{cl}_{M(\mathcal{C})}(X)$, if $\mathrm{cl}_{M(\mathcal{C})}(X) \neq X$, then $\mathrm{cl}_{M(\mathcal{C})}(X) \nsubseteq X$, that is, there exists $y \notin X$ such that $r_{M(\mathcal{C})}(X)=r_{M(\mathcal{C})}(X \cup\{y\})$. Suppose $T=\left\{t_{1}, t_{2}, \ldots, t_{t}\right\}(t \leq s)$ is a maximal independent set included in $X$, then $\left\{t_{1}, t_{2}, \ldots, t_{t}\right\} \subseteq X=$ $\bigcup_{x \in X} I(x)$ and there exist different $K_{1}, K_{2}, \ldots, K_{t}$ such that for all $i \in\{1,2, \ldots, t\}, t_{i} \in K_{i}$. Since $y \notin X, I(x) \cap I(y)=\emptyset$ for all $x \in X$. Based on $\left\{I\left(x_{1}\right), I\left(x_{2}\right), \ldots, I\left(x_{s}\right)\right\}$ forms a partition of $E$, there exists $K \subseteq I(y)$ such that $K_{1}, K_{2}, \ldots, K_{t}, K$ are different blocks and $y \in K$, thus $T \cup\{y\}$ is a maximal independent set included in $X \cup\{y\}$. Hence, we have $r_{M(\mathcal{C})}(X \cup\{y\})=r_{M(\mathcal{C})}(X)+1$ which contradicts $r_{M(\mathcal{C})}(X)=r_{M(\mathcal{C})}(X \cup\{y\})$. Thus we can obtain $\mathrm{cl}_{M(\mathcal{C})}(X)=X$.

The following proposition illustrates that in what condition the indiscernible neighborhoods are included in the geometric lattice induced by $\mathcal{C}$.

Proposition 5.2. Let $\mathcal{C}$ be a covering of $E$. If $S H$ induced by $\mathcal{C}$ is a closure operator, then for all $x \in E, I(x) \in \mathcal{L}(M(\mathcal{C}))$.

Proof. Since SH induced by $C$ is a closure operator, $\{I(x): x \in E\}$ forms a partition of $E$. Thus, for all $x \in E, \operatorname{SH}(I(x))=\bigcup_{y \in I(x)} I(y)=I(x)$, that is, $I(x) \in \mathcal{L}_{\mathrm{SH}}(M(\mathcal{C}))$. According to Proposition 5.1, $I(x) \in \mathcal{L}(M(\mathcal{C}))$.

We give an example to help understand the relationship between $\mathcal{L}_{\mathrm{SH}}(M(\mathcal{C}))$ and $\mathcal{L}(M(\mathcal{C}))$ better.

Example 5.3. Let $\mathcal{C}=\{\{1,2\},\{1,3\},\{2,3\},\{4,5\}\} . I(1)=I(2)=I(3)=\{1,2,3\}$, $I(4)=I(5)=\{4,5\}$. Let $T=\{1,2\} . T \in \supset(\mathcal{C})$ but $T \notin \supset_{\mathrm{SH}}(\mathcal{C})$ because $|T \cap I(1)|=2$. Hence, $\supset(\mathcal{C}) \nsubseteq \supset_{\mathrm{SH}}(\mathcal{C}) . \mathcal{L}_{\mathrm{SH}}(M(\mathcal{C}))=\{\emptyset,\{1,2,3\},\{4,5\},\{1,2,3,4,5\}\}$. $\mathcal{L}(M(\mathcal{C}))=\{\emptyset,\{1\},\{2\},\{3\},\{4,5\},\{1,2\},\{1,3\},\{2,3\},\{1,4,5\},\{2,4,5\},\{3,4,5\},\{1,2,3\}$, $\{1,2,4,5\},\{1,3,4,5\},\{2,3,4,5\},\{1,2,3,4,5\}\}$. It is obvious that $\mathcal{L}(M(\mathcal{C})) \nsubseteq \mathcal{L}_{\mathrm{SH}}(M(\mathcal{C}))$. The structures of $\mathcal{L}_{\mathrm{SH}}(M(\mathcal{C}))$ and $\mathcal{L}(M(\mathcal{C}))$ are showed in Figure 1. 

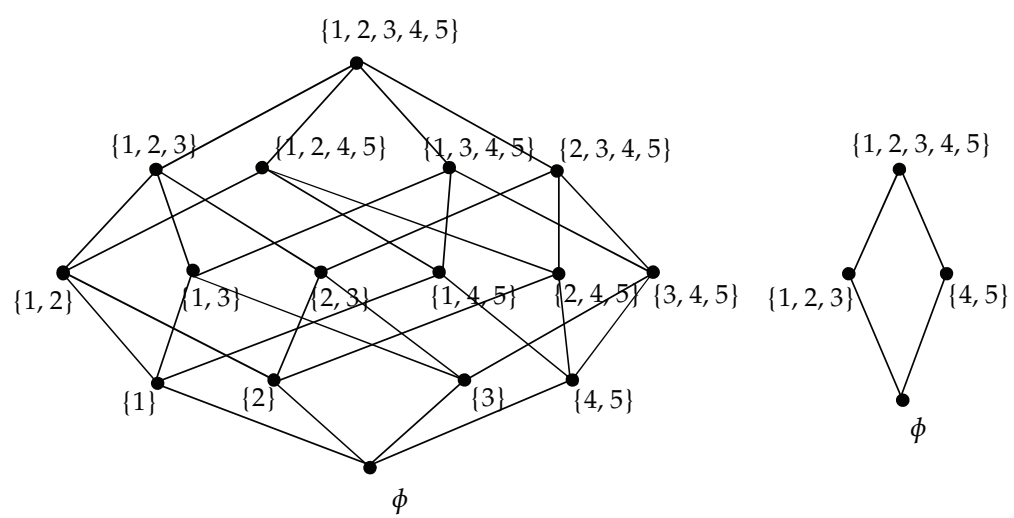

Figure 1: The lattice of $\mathcal{\perp}(M(\mathcal{C}))\left(\right.$ resp., $\left.\mathcal{L}_{\mathrm{XH}}(M(\mathcal{C}))\right)$ and $\mathcal{L}_{\mathrm{SH}}(M(\mathcal{C}))$.

Remark 5.4. Let $\mathcal{C}$ be a covering of $E$. Although $\mathrm{XH}$ induced by $\mathcal{C}$ is a closure operator, it has no relationship between $\supset_{\mathrm{XH}}(\mathcal{C})$ and $\mathcal{\supset}(\mathcal{C})$, and has no relationship between $\mathcal{L}_{\mathrm{XH}}(M(\mathcal{C}))$ and $\mathcal{L}(M(\mathcal{C}))$.

The following example illustrates the above statements.

Example 5.5. Let $\mathcal{C}=\left\{K_{1}, K_{2}, K_{3}, K_{4}\right\}$ be a covering of $E=\{a, b, c, d, e, f, g, h, i\}$, where $K_{1}=$ $\{a, b, i\}, K_{2}=\{a, b, c, d, e, f\}, K_{3}=\{f, g, h\}, K_{4}=\{c, d, e, g, h, i\}$. Then $N(a)=N(b)=$ $\{a, b\}, N(c)=N(d)=N(e)=\{c, d, e\}, N(f)=\{f\}, N(g)=N(h)=\{g, h\}$ and $N(i)=\{i\}$. Let $T=\{a, c, f, g, i\}$. It is clear that $T \in \supset_{\mathrm{XH}}(\mathcal{C})$, but $T \notin \supset(\mathcal{C})$ because $\left|T \cap K_{2}\right|=2$, thus $\supset_{\mathrm{XH}}(\mathcal{C}) \nsubseteq \supset(\mathcal{C})$. Let $T^{\prime}=\{a, \mathcal{C}, d\}$. It is clear that $T^{\prime} \in \supset(\mathcal{C})$, but $T^{\prime} \notin \supset_{\mathrm{XH}}$ because $\left|T^{\prime} \cap N(c)\right|=$ 2 , thus $\supset(\mathcal{C}) \nsubseteq \supset_{\mathrm{XH}}(\mathcal{C})$. Let $X=\{a, b, i\} . X \in \mathcal{L}_{\mathrm{XH}}(M(\mathcal{C}))$ for $\mathrm{XH}(X)=X$. However, $X \notin$ $\mathcal{L}(M(\mathcal{C}))$ for $\mathrm{cl}_{M(\mathcal{C})}(X)=\{a, b, c, d, e, i\}$. Let $X=\{a\}$. $X \in \mathcal{L}(M(\mathcal{C}))$ for $\mathrm{cl}_{M(\mathcal{C})}(X)=X$. However, $X \notin \mathcal{L}_{\mathrm{XH}}(M(\mathcal{C}))$ for $\mathrm{XH}(X)=\{a, b\} \neq X$.

The following proposition shows the relationship between $\supset_{\mathrm{SH}}(\mathcal{C})$ and $\supset_{\mathrm{XH}}(\mathcal{C})$, and the relationship between $\mathcal{L}_{\mathrm{SH}}(M(\mathcal{C}))$ and $\mathcal{L}_{\mathrm{XH}}(M(\mathcal{C}))$.

Proposition 5.6. Let $\mathcal{C}$ be a covering of $E$. If XH and SH induced by $\mathcal{C}$ are closure operators, then $\supset_{S H}(\mathcal{C}) \subseteq \supset_{X H}(\mathcal{C})$ and $\mathcal{L}_{S H}(M(\mathcal{C})) \subseteq \mathcal{L}_{X H}(M(\mathcal{C}))$.

Proof. If XH and SH induced by $\mathcal{C}$ are closure operators, then $\{N(x): x \in E\}$ and $\{I(x): x \in$ $E$ \} form a partition of $E$, respectively. For all $x \in X, N(x)=\bigcap_{x \in K} K \subseteq \bigcup_{i \in K} K=I(x)$, thus $\{N(x): x \in E\}$ is finer than $\{I(x): x \in E\}$. Based on this, we can obtain $\supset_{\mathrm{SH}}(\mathcal{C}) \subseteq \supset_{\mathrm{XH}}(\mathcal{C})$.

For all $X \in \mathcal{L}_{\mathrm{SH}}(M(\mathcal{C})), X=\mathrm{SH}(X)=\bigcup_{x \in X} I(x)$. Since $x \in N(x) \subseteq I(x), X=$ $\bigcup_{x \in X}\{x\} \subseteq \bigcup_{x \in X} N(x) \subseteq \bigcup_{x \in X} I(x)=X$, thus, $X=\bigcup_{x \in X} N(x)$, that is, $X \in \mathcal{L}_{\mathrm{XH}}(M(\mathcal{C}))$. Hence, $\mathcal{L}_{\mathrm{SH}}(M(\mathcal{C})) \subseteq \mathcal{L}_{\mathrm{XH}}(M(\mathcal{C}))$.

Example 5.7. From Example 5.3, we know $N(1)=\{1\}, N(2)=\{2\}, N(3)=\{3\}$, $N(4)=N(5)=\{4,5\}$ and $I(1)=I(2)=I(3)=\{1,2,3\}, I(4)=I(5)=\{4,5\}$. Let $I=\{1,2\}$. It is clear that $I \in \supset_{\mathrm{XH}}(M(\mathcal{C}))$ but $I \notin \supset_{\mathrm{SH}}(M(\mathcal{C}))$ because $\mid I \cap$ $I(1) \mid=2$. Hence $\supset_{\mathrm{XH}}(M(\mathcal{C})) \nsubseteq \supset_{\mathrm{SH}}(M(\mathcal{C})) . \mathcal{L}_{\mathrm{SH}}(M(\mathcal{C}))=\{\emptyset,\{1,2,3\},\{4,5\},\{1,2,3,4,5\}\}$ and $\mathcal{L}_{\mathrm{XH}}(M(\mathcal{C}))=\{\emptyset,\{1\},\{2\},\{3\},\{4,5\},\{1,2\},\{1,3\},\{2,3\},\{1,4,5\},\{2,4,5\},\{3,4,5\},\{1,2,3\}$, 
Journal of Applied Mathematics

$\{1,2,4,5\},\{1,3,4,5\},\{2,3,4,5\},\{1,2,3,4,5\}\}$. It is obvious that $\mathcal{L}_{\mathrm{XH}}(M(\mathcal{C})) \nsubseteq \mathcal{L}_{\mathrm{SH}}(M(\mathcal{C}))$. The structures of them are showed in Figure 1.

When a covering degenerates into a partition, we can obtain the following result.

Theorem 5.8. If $\mathcal{C}$ is a partition of $E$, then $\supset_{S H}(\mathcal{C})=\supset_{X H}(\mathcal{C})=\supset(\mathcal{C})$ and $\mathcal{L}_{S H}(M(\mathcal{C}))=$ $\mathcal{L}_{\mathrm{XH}}(M(\mathcal{C}))=\mathcal{L}(M(\mathcal{C}))$.

Proof. Since $C$ is a partition of $E, I(x)=N(x)=K(x \in K)$ for all $x \in E$, and for all $X \subseteq E$, $\mathrm{SH}(X)=\mathrm{XH}(X)=R^{*}(X)$. Thus $\supset_{\mathrm{SH}}(\mathcal{C})=\varrho_{\mathrm{XH}}(\mathcal{C})=\mathcal{I}(\mathcal{C})$ and $\mathcal{L}_{\mathrm{SH}}(M(\mathcal{C}))=\mathcal{L}_{\mathrm{XH}}(M(\mathcal{C}))=$ $\mathcal{L}(M(\mathcal{C}))$.

Next, we discuss the reducible element and immured element's influence on matroidal structures and geometric lattice structures. First, we study the reducible element and immured element's influence on $\supset(\mathcal{C})$.

Theorem 5.9. Let $\mathcal{F}$ be a family of subset of $E$ and $K \in \mathcal{F} . \supset(\mathcal{F}-\{K\}) \subseteq \supset(\mathcal{F})$.

Proof. For all $I \in \supset(\mathcal{F}-\{K\})$, we may as well suppose $I=\left\{i_{1}, i_{2}, \ldots, i_{t}\right\}$ where $i_{1}, i_{2}, \ldots, i_{t} \in E$. According to the definition of transversal matroid, there exist different blocks $K_{1}, K_{2}, \ldots, K_{t} \in$ $\mathcal{F}$ satisfy $K_{i} \neq K$ and $i_{j} \in K_{j}$ for all $1 \leq i, j \leq t$. Thus $I \in \mathcal{I}(\mathcal{F})$.

The following example illustrates $\supset(\mathcal{F}) \nsubseteq \supset(\mathcal{F}-\{K\})$.

Example 5.10. Let $\mathcal{F}=\left\{K_{1}, K_{2}, K_{3}\right\}$ be a family of subset of $E=\{1,2,3,4\}$, where $K_{1}=\{1,2\}$, $K_{2}=\{1,3\}, K_{3}=\{3\} . \supset(\mathcal{F})=2^{E}, \supset\left(\mathcal{F}-\left\{K_{3}\right\}\right)=\{\emptyset,\{1\},\{2\},\{3\},\{1,3\},\{1,2\}\{2,3\}\}$. Hence, $\supset(\mathscr{F}) \nsubseteq \supset(\mathscr{F}-\{K\})$.

Let $\mathcal{C}$ be a covering of $E$ and $\mathcal{F}=\mathcal{C}$. As we know, reducible elements and immured elements are members of $\mathcal{C}$. Based on Theorem 5.9, it is not difficult for us to obtain the following four corollaries.

Corollary 5.11. Let $\mathcal{C}$ be a covering of $E$ and $K \in \mathcal{C}$. If $K$ is reducible, then $\supset(\mathcal{C}-\{K\}) \subseteq \supset(\mathcal{C})$.

Corollary 5.12. Let $\mathcal{C}$ be a covering of $E . \supset(\operatorname{reduct}(\mathcal{C})) \subseteq \supset(\mathcal{C})$.

Corollary 5.13. Let $\mathcal{C}$ be a covering of $E$ and $K \in \mathcal{C}$. If $K$ is an immured element, then $\supset(\mathcal{C}-\{K\}) \subseteq$ $\supset(\mathcal{C})$

Corollary 5.14. Let $\mathcal{C}$ be a covering of $E . \supset(\operatorname{exclusion}(\mathcal{C})) \subseteq \supset(\mathcal{C})$.

The following theorem shows the reducible element and immured element's influence on geometric lattice structure $\mathcal{L}(M(\mathcal{C}))$.

Theorem 5.15. Let $\mathscr{F}$ be a family of subset of $E$ and for all $K \in \mathcal{F} . \mathcal{L}(M(\mathcal{F}-\{K\})) \subseteq \mathcal{L}(M(\mathscr{F}))$.

Proof. First, we prove $\mathrm{cl}_{\mathcal{F}}(\{x\}) \subseteq \mathrm{cl}_{\mathcal{F}-\{K\}}(\{x\})$ for all $x \in E$. For all $y \notin \mathrm{cl}_{\mathcal{F}-\{K\}}(\{x\}),\{x, y\} \in$

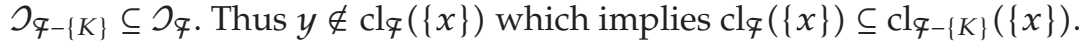

Second, we need to prove that any atom of $\mathcal{L}(M(\mathcal{F}-\{K\}))$ is a closed set of $\mathcal{L}(M(\mathcal{F}))$, that is, $\operatorname{cl}_{\mathcal{F}}\left(\operatorname{cl}_{\mathcal{F}-\{K\}}(\{x\})\right)=\operatorname{cl}_{\mathcal{F}-\{K\}}(\{x\})$. Since $\mathrm{cl}_{\mathcal{F}-\{K\}}(\{x\}) \subseteq \operatorname{cl}_{\mathcal{F}}\left(\operatorname{cl}_{\mathcal{F}-\{K\}}(\{x\})\right) \subseteq$

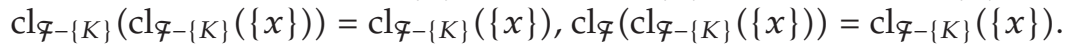


Third, we need to prove $\mathcal{L}(M(\mathcal{F}-\{K\})) \subseteq \mathcal{L}(M(\mathcal{F}))$. For all $X \subseteq \mathcal{L}(M(\mathcal{F}-\{K\}))$, $\mathrm{X}=\bigvee_{i=1}^{m} \mathrm{cl}_{\mathcal{F}-\{K\}}(\{x\})=\bigvee_{i=1}^{m} \bigvee_{j=1}^{s_{i}} \mathrm{cl}_{\mathcal{F}}\left(\left\{x_{j}\right\}\right)$ because $\mathcal{L}(M(\mathcal{F}-\{K\}))$ is a atomic lattice, thus $X \in \mathcal{L}(M(\mathcal{F}))$.

The following example shows that $\mathcal{L}(M(\mathcal{F})) \nsubseteq \mathcal{L}(M(\mathcal{F}-\{K\}))$, where $K \in \mathcal{F}$.

Example 5.16. Based on Example 5.10, we have $\mathcal{L}(M(\mathcal{F}))=2^{E}$ and $\mathcal{L}\left(M\left(\mathcal{F}-\left\{K_{3}\right\}\right)\right)=$ $\{\emptyset,\{1\},\{2\},\{3\},\{1,2,3\}\}$. It is clear that $\mathcal{L}(M(\mathcal{F})) \nsubseteq \mathcal{L}\left(M\left(\mathcal{F}-\left\{K_{3}\right\}\right)\right)$.

Similarly, when $\mathcal{F}$ is equal to $\mathcal{C}$, we can obtain the following four corollaries.

Corollary 5.17. Let $\mathcal{C}$ be a covering of $E$ and $K \in \mathcal{C}$. If $K$ is reducible, then $\mathcal{L}(M(\mathcal{C}-\{K\})) \subseteq$ $\mathcal{L}(M(\mathcal{C}))$.

Corollary 5.18. Let $\mathcal{C}$ be a covering of $E . \mathcal{L}(M(\operatorname{reduct}(\mathcal{C}))) \subseteq \mathcal{L}(M(\mathcal{C}))$.

Corollary 5.19. Let $\mathcal{C}$ be a covering of $E$ and $K \in \mathcal{C}$. If $K$ is an immured element, then $\mathcal{L}(M(\mathcal{C}-$ $\{K\})) \subseteq \mathcal{L}(M(\mathcal{C}))$.

Corollary 5.20. Let $\mathcal{C}$ be a covering of $E . \mathcal{L}(M($ exclusion $(\mathcal{C}))) \subseteq \mathcal{L}(M(\mathcal{C}))$.

Let $\mathcal{C}$ be a covering of $E$ and $\mathrm{SH}$ induced by $\mathcal{C}$ a closure operator. If a reducible element $K$ of $\mathcal{C}$ is removed from the covering $\mathcal{C}$, then covering $\mathcal{C}-\{K\}$ may not still be a covering which makes $\mathrm{SH}$ be a closure operator. Hence, we omit the discussion of the relationship between $\supset_{\mathrm{SH}}(\mathrm{C})$ and $\supset_{\mathrm{SH}}(\mathrm{C}-\{K\})$.

Example 5.21. Let $E=\{1,2,3\}$ and $\mathcal{C}=\left\{K_{1}, K_{2}, K_{3}\right\}$ where $K_{1}=\{1,2\}, K_{2}=\{1,3\}, K_{3}=$ $\{1,2,3\} . I(1)=I(2)=I(3)=\{1,2,3\}$, thus $\{I(x): x \in E\}$ forms a partition of $E$. Hence, $\mathrm{SH}$ is a closure operator induced by $\mathcal{C}$. It is clear that $K_{3}$ is a reducible element, $\mathcal{C}-\left\{K_{3}\right\}=\left\{K_{1}, K_{2}\right\}$. Then the indiscernible neighborhoods induced by $\mathcal{C}-\left\{K_{3}\right\}$ are $I(1)=\{1,2,3\}, I(2)=\{1,2\}$, $I(3)=\{1,3\}$. we find that $\{I(x): x \in E\}$ cannot form a partition of $E$. Hence, SH is not a closure operator induced by $\mathcal{C}-\left\{K_{3}\right\}$.

The following theorem presents an immured element's influence on $\supset_{\mathrm{SH}}(\mathcal{C})$ and $\left\llcorner_{\mathrm{SH}}(M(\mathcal{C}))\right.$.

Theorem 5.22. Let $\mathcal{C}$ be a covering of $E$ and $K$ an immured element of $\mathcal{C}$. If $S H$ induced by $\mathcal{C}$ is a closure operator, then $S H$ induced by covering $\mathcal{C}-\{K\}$ is also a closure operator. Moreover, $\supset_{S H}(\mathcal{C})=$ $\supset_{S H}(\mathcal{C}-\{K\})$ and $\mathcal{L}_{S H}(M(\mathcal{C}))=\mathcal{L}_{S H}(M(\mathcal{C}-\{K\}))$.

Proof. It comes from Lemma 4.8 and Theorem 4.3.

As we know, if $\mathcal{C}$ is a covering of $E, K_{1}$ and $K_{2}$ are two elements of $\mathcal{C}$, and $K_{1}$ is an immured element of $\mathcal{C}$, then $K_{2}$ is an immured element of $\mathcal{C}$ if and only if $K_{2}$ is an immured element of the covering $\mathcal{C}-\left\{K_{1}\right\}$. Combining this with Theorem 5.22, we can obtain the following corollary.

Corollary 5.23. Let $\mathcal{C}$ be a covering of $E$. If SH induced by $\mathcal{C}$ is a closure operator, then $S H$ induced by exclusion $(\mathcal{C})$ is also a closure operator. Moreover, $\supset_{S H}(\mathcal{C})=\supset_{S H}(\operatorname{exclusion}(\mathcal{C}))$ and $\perp_{S H}(M(\mathcal{C}))=$ $\mathcal{L}_{S H}(M(\operatorname{exclusion}(\mathcal{C})))$.

Now we consider the reducible element's influence on $\supset_{\mathrm{XH}}(\mathcal{C})$ and $\mathcal{L}_{\mathrm{XH}}(M(\mathcal{C}))$. 
Theorem 5.24. Let $\mathcal{C}$ be a covering of $E$ and $K$ be a reducible element. If XH induced by $C$ is a closure operator, then $\mathrm{XH}$ induced by covering $\mathcal{C}-\{K\}$ is also a closure operator. Moreover, $\supset_{\mathrm{XH}}(\mathcal{C})=$ $\supset_{\mathrm{XH}}(\mathcal{C}-\{K\})$ and $\mathcal{\perp}_{\mathrm{XH}}(M(\mathcal{C}))=\mathcal{L}_{\mathrm{XH}}(M(\mathcal{C}-\{K\}))$.

Proof. Since $\mathrm{XH}$ induced by $\mathcal{C}$ is a closure operator, $\{N(x): x \in E\}$ induced by $\mathcal{C}$ forms a partition. Based on the definition of $\supset_{\mathrm{XH}}(\mathcal{C})$ and Lemma $4.18, \mathrm{XH}$ induced by $\mathcal{C}-\{K\}$ is also a closure operator and $\supset_{\mathrm{XH}}(\mathcal{C})=\supset_{\mathrm{XH}}(\mathcal{C}-\{K\})$.

As we know, if $\mathcal{C}$ is a covering of $E, K \in \mathcal{C}, K$ is reducible in $\mathcal{C}$ and $K_{1} \in \mathcal{C}-\{K\}$, then $K_{1}$ is reducible in covering $\mathcal{C}$ if and only if it is reducible in covering $\mathcal{C}-\{K\}$. Based on this and Theorem 5.24, we can obtain the following result.

Corollary 5.25. Let $\mathcal{C}$ be a covering of $E$. If XH induced by $\mathcal{C}$ is a closure operator, then $X H$ induced by reduct $(\mathcal{C})$ is a closure operator. Moreover, $\supset_{X H}(\mathcal{C})=\supset_{X H}($ reduct $(\mathcal{C}))$ and $\mathcal{L}_{X H}(M(\mathcal{C}))=$ $\mathcal{L}_{\mathrm{XH}}(\mathrm{M}(\operatorname{reduct}(\mathcal{C})))$.

Let $\mathcal{C}$ be a covering of $E$ and $\mathrm{XH}$ induced by $\mathcal{C}$ a closure operator. If an immured element $K$ is removed from the covering $\mathcal{C}$, then $\mathcal{C}-\{K\}$ may not still be a covering which makes $\mathrm{XH}$ be a closure operator. So we omit the discussion of the relationship between $\supset_{\mathrm{XH}}(\mathcal{C})$ and $\supset_{\mathrm{XH}}(\mathcal{C}-\{K\})$.

Example 5.26. Suppose $K_{1}=\{1\}, K_{2}=\{1,2\}, K_{3}=\{2,3\}, K_{4}=\{3\}, K_{5}=\{1,2,3\}$ and $\mathcal{C}_{1}=\left\{K_{1}, K_{2}, K_{3}, K_{4}\right\}$. Then $N(1)=\{1\}, N(2)=\{2\}$ and $N(3)=\{3\}$, thus $\{N(x): x \in E\}$ forms a partition of $E$. Hence, $\mathrm{XH}$ is a closure operator. It is clear that $K_{1}$ is an immured element, and the neighborhoods induced by $\mathcal{C}-\left\{K_{1}\right\}$ are $N(1)=\{1,2\}, N(2)=\{2\}$ and $N(3)=\{3\}$, thus $\{N(x): x \in E\}$ cannot form a partition of $E$. Hence, $\mathcal{C}-\left\{K_{1}\right\}$ is not a covering which makes $\mathrm{XH}$ be a closure operator.

\section{Conclusions}

This paper has studied the geometric lattice structures of covering based-rough sets through matroids. The important contribution of this paper is that we have established a geometric lattice structure of covering-based rough sets through the transversal matroid induced by a covering and have presented two geometric lattice structures of covering-based rough sets through two types of covering upper approximation operators. Moreover, we have discussed the relationship among the three geometric lattice structures. To study other properties of the geometric lattice structure induced by a covering and to study other geometric lattices from the viewpoint of other upper approximation operators are our future work.

\section{Acknowledgments}

This work is supported in part by the National Natural Science Foundation of China under Grant no. 61170128, the Natural Science Foundation of Fujian Province, China, under Grant nos. 2011J01374 and 2012J01294, and the Science and Technology Key Project of Fujian Province, China, under Grant no. 2012H0043.

\section{References}

[1] Z. Pawlak, "Rough sets," International Journal of Computer and Information Sciences, vol. 11, no. 5, pp. 341-356, 1982. 
[2] A. Skowron and J. Stepaniuk, "Tolerance approximation spaces," Fundamenta Informaticae, vol. 27, no. 2-3, pp. 245-253, 1996.

[3] R. Slowinski and D. Vanderpooten, "A generalized definition of rough approximations based on similarity," IEEE Transactions on Knowledge and Data Engineering, vol. 12, no. 2, pp. 331-336, 2000.

[4] G. Liu and W. Zhu, "The algebraic structures of generalized rough set theory," Information Sciences, vol. 178, no. 21, pp. 4105-4113, 2008.

[5] Y. Y. Yao, "On generalizing pawlak approximation operators," in Proceedings of the 1st International Conference on Rough Sets and Current Trends in Computing (RSCTC '98), vol. 1424 of Lecture Notes in Artificial Intelligence, pp. 298-307, 1998.

[6] Y. Y. Yao, "Relational interpretations of neighborhood operators and rough set approximation operators," Information Sciences, vol. 111, no. 1-4, pp. 239-259, 1998.

[7] Y. Y. Yao, "Constructive and algebraic methods of the theory of rough sets," Information Sciences, vol. 109, no. 1-4, pp. 21-47, 1998.

[8] W. Zhu and F. Wang, "A new type of covering rough sets," in Proceedings of IEEE International Conference on Intelligent Systems, pp. 444-449, London, UK, September 2006.

[9] K. Qin, Y. Gao, and Z. Pei, "On covering rough sets," in Proceedings of the 2 nd International Conference on Rough Sets and Knowledge Technology (RSKT '07), vol. 4481 of Lecture Notes in Computer Science, pp. 34-41, 2007.

[10] S. Wang, P. Zhu, and W. Zhu, "Structure of covering-based rough sets," International Journal of Mathematical and Computer Sciences, vol. 6, pp. 147-150, 2010.

[11] S. Wang, Q. Zhu, W. Zhu, and F. Min, “Quantitative analysis for covering-based rough sets through the upper approximation number," Information Sciences, vol. 220, pp. 483-491, 2013.

[12] L. Wang, X. Yang, J. Yang, and C. Wu, "Relationships among generalized rough sets in six coverings and pure reflexive neighborhood system," Information Sciences, vol. 207, pp. 66-78, 2012.

[13] Y. Yao and B. Yao, "Covering based rough set approximations," Information Sciences, vol. 200, pp. 91-107, 2012.

[14] L. Wang, X. Liu, and J. Cao, "A new algebraic structure for formal concept analysis," Information Sciences, vol. 180, no. 24, pp. 4865-4876, 2010.

[15] R. Wille, "Restructuring lattice theory: an approach based on hierarchies of concepts," in Ordered Sets, I. Rival, Ed., pp. 445-470, Reidel, Dordrecht, The Netherlands, 1982.

[16] Y. Y. Yao and Y. Chen, "Rough set approximations in formal concept analysis," in Proceedings of the 23rd International Meeting of the North American Fuzzy Information Processing Society, pp. 73-78, June 2004.

[17] G. Birhoff, Lattice Theory, American Mathematical Society, Rhode Island, RI, USA, 1995.

[18] H. Lai, Matroid Theory, Higher Education Press, Beijing, China, 2001.

[19] J. G. Oxley, Matroid Theory, Oxford University Press, New York, NY, USA, 1993.

[20] J. Edmonds, "Matroids and the greedy algorithm," Mathematical Programming, vol. 1, pp. 127-136, 1971.

[21] E. Lawler, Combinatorial Optimization: Networks and Matroids, Dover Publications, 2001.

[22] S. Wang and W. Zhu, "Matroidal structure of covering-based rough sets through the upper approximation number," International Journal of Granular Computing, Rough Sets and Intelligent Systems, vol. 2, pp. 141-148, 2011.

[23] S. Wang, W. Zhu, and F. Min, "Transversal and function matroidal structures of covering-based rough sets," in Proceedings of the 6th International Conference on Rough Sets and Knowledge Technology (RSKT '11), pp. 146-155, 2011.

[24] S. Wang, Q. Zhu, W. Zhu, and F. Min, "Matroidal structure of rough sets and its characterization to attribute reduction," Knowledge-Based Systems, vol. 36, pp. 155-161, 2012.

[25] W. Zhu and S. Wang, "Matroidal approaches to generalized rough sets based on relations," International Journal of Machine Learning and Cybernetics, vol. 2, no. 4, pp. 273-279, 2011.

[26] W. Zhu, "Relationship between generalized rough sets based on binary relation and covering," Information Sciences, vol. 179, no. 3, pp. 210-225, 2009.

[27] W. Zhu and F. Wang, "On three types of covering rough sets," IEEE Transactions on Knowledge and Data Engineering, vol. 19, pp. 1131-1144, 2007.

[28] W. Zhu, "Topological approaches to covering rough sets," Information Sciences, vol. 177, no. 6, pp. 1499-1508, 2007.

[29] W. Zhu and F.-Y. Wang, "Reduction and axiomization of covering generalized rough sets," Information Sciences, vol. 152, pp. 217-230, 2003. 
[30] J. A. Pomykała, "Approximation operations in approximation space," Bulletin of the Polish Academy of Sciences, vol. 35, no. 9-10, pp. 653-662, 1987.

[31] Z. Y. Xu and Q. Wang, "Properties of covering rough sets model," Journal of Henan Normal University, vol. 33, no. 1, pp. 130-132, 2005.

[32] W. Żakowski, "Approximations in the space $(u, \pi)$, " Demonstratio Mathematica, vol. 16, no. 3, pp. 761$769,1983$.

[33] Y.-L. Zhang, J. Li, and W.-Z. Wu, "On axiomatic characterizations of three pairs of covering based approximation operators," Information Sciences, vol. 180, no. 2, pp. 274-287, 2010.

[34] G. Hu, X. Xiao, and W. Zhang, "Study on conditions of neighborhoods forming a partition," in Proceedings of the 9th International Conference on Fuzzy Systems and Knowledge Discovery (FSKD '12), pp. 256-259, May 2012.

[35] Z. Yun, X. Ge, and X. Bai, "Axiomatization and conditions for neighborhoods in a covering to form a partition," Information Sciences, vol. 181, no. 9, pp. 1735-1740, 2011. 


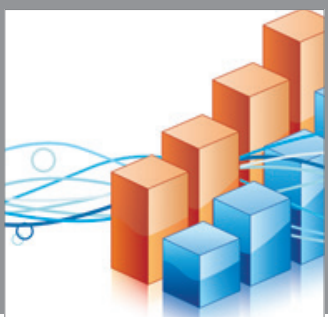

Advances in

Operations Research

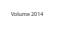

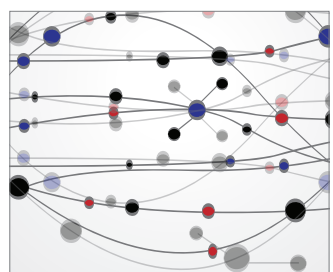

\section{The Scientific} World Journal
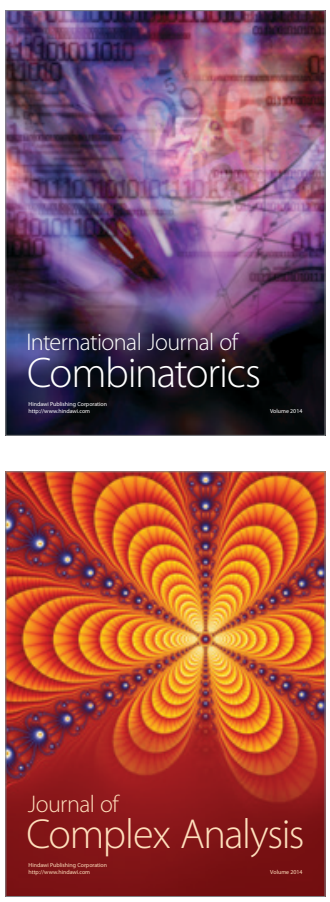

International Journal of

Mathematics and

Mathematical

Sciences
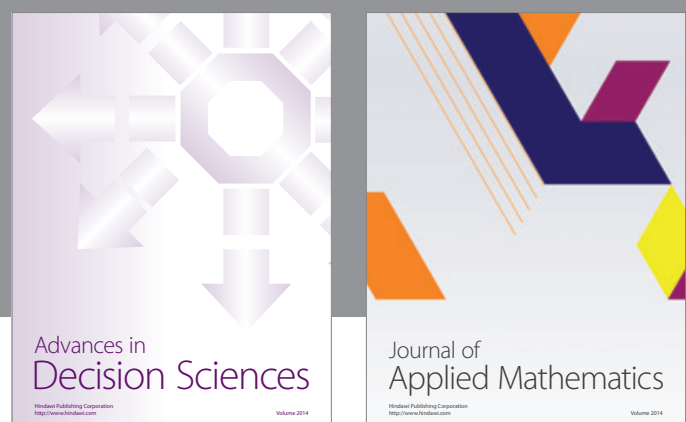

Journal of

Applied Mathematics
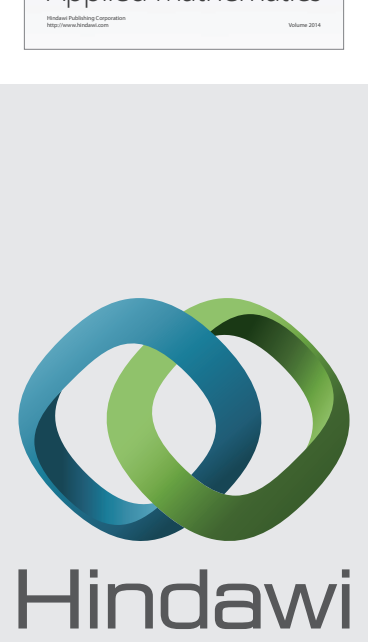

Submit your manuscripts at http://www.hindawi.com
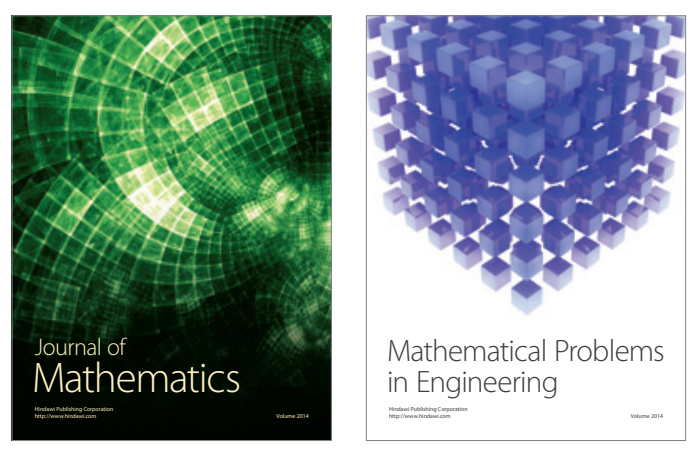

Mathematical Problems in Engineering
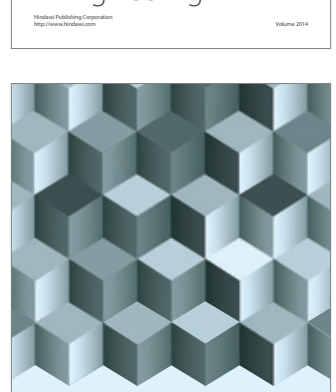

Journal of

Function Spaces
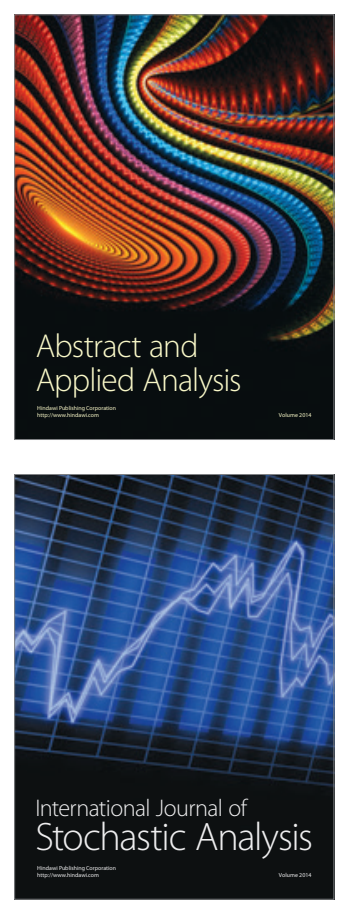

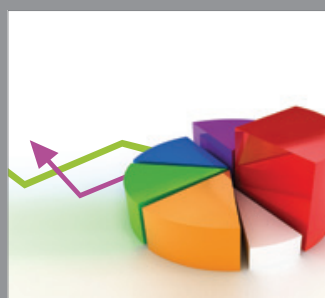

ournal of

Probability and Statistics

Promensencen
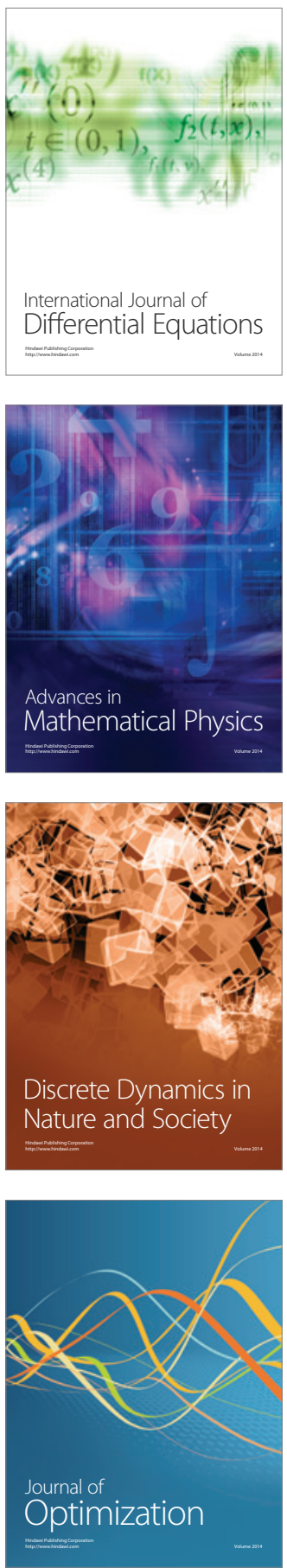